Muñoz Cantero, J.M., Casar Domínguez, L. S. y Abalde Paz, E. (2007). El “contexto" y las "metas y objetivos" como elementos clave en la calidad de la atención a la diversidad en centros no universitarios.

RELIEVE, v. 13, n. 2, p. 235-261. http://www.uv.es/RELIEVE/v13n2/RELIEVEv13n2 6.htm

\title{
REIIदVE
}

Revista ELectrónica de Investigación

y EValuación Educativa

\section{EL “CONTEXTO Y LAS “METAS Y OBJETIVOS” COMO ELEMENTOS CLAVE EN LA CALIDAD DE LA ATENCIÓN A LA DIVERSIDAD EN CENTROS NO UNIVERSITARIOS}

\section{[The "context" and "goals and objectives" as key elements in quality in attention to diversity in non-university education]}

Article record

$\underline{\text { About authors }}$

HTML format

\author{
Muñoz Cantero, Jesús Miguel (munoz@udc.es) \\ Casar Domínguez, Luz Sandra (sandracasar@edu.xunta.es) \\ Abalde Paz, Eduardo (abalde@udc.es)
}

Ficha del artículo

$\underline{\text { Sobre los autores }}$

Formato HTML

\begin{abstract}
Currently there is a growing interest in educational quality and attention to diversity. This paper presents an analysis of two important aspects: the "context" in which the school must develop its activity and the "goals and objectives" that a school should respond to define quality for diversity. Analyze the degree of importance for teachers and the level of compliance in the centers, from data obtained in two of the questionnaires. The study was conducted with teachers in the province of A Coruna (Spain). Overall, we can say that the issues explored are considered important by teachers Las indicators of quality. However, on the contrary, its level of compliance in the centers is medium or low, forcing establishing measures for improvement.
\end{abstract}

\section{Keywords}

Quality, attention to diversity, inclusion, testing, nonuniversity education

\begin{abstract}
Resumen
Actualmente existe un interés creciente por la calidad educativa y la atención a la diversidad. En este trabajo se presenta un análisis de dos aspectos importantes: el "Contexto" en el que el centro debe de desarrollar su actividad y las "metas y objetivos" que un centro educativo debe definir para dar una respuesta de calidad a la diversidad. Analizaremos el grado de importancia para los profesores y el nivel de cumplimiento en los centros, a partir de los datos obtenidos en dos cuestionarios. El estudio se realizó con profesores de la provincia de A Coruña (España). En líneas generales podemos decir que los aspectos analizados son considerados importantes por los profesores como indicadores de calidad. Sin embargo, por el contrario, su nivel de cumplimiento en los centros es medio o bajo, lo que obliga a establecer medidas de mejora y correctores de estrategias.
\end{abstract}

\section{Descriptores}

Calidad, atención a la diversidad, inclusión, evaluación, educación no universitaria.

\section{Introducción}

Que las personas difieren en sus características es aceptado mayoritariamente, pero sólo en los últimos años se ha visto la necesidad de una mejor y más justa atención: Una educación de calidad que incluya a todos. "La actitud por si sola no basta para crear 
Muñoz Cantero, J.M., Casar Domínguez, L. S. y Abalde Paz, E. (2007). El “contexto" y las "metas y objetivos" como elementos clave en la calidad de la atención a la diversidad en centros no universitarios.

RELIEVE, v. 13, n. 2, p. 235-261. http://www.uv.es/RELIEVE/v13n2/RELIEVEv13n2 6.htm

las condiciones necesarias para la inclusión, también es cierto que sin una actitud favorable poco se podrá avanzar. La educación inclusiva implica un cambio de mentalidad. Una nueva forma de pensar acerca de las diferencias $y$, por supuesto, una nueva forma de entender la educación” (Cardona, 2006: 202). En este trabajo pretendemos hacer una reflexión sobre dos factores estrechamente relacionados que determinan una atención de calidad hacia la diversidad: el "contexto" y las "metas y objetivos" que un centro educativo tiene a la hora de hacer frente a su diversidad.

Se reconoce la diversidad como el resultado de la interacción de un conjunto de dimensiones diferenciadoras (capacidad, motivación, intereses, la historia académica y expectativas) que explican las diferencias en el aprendizaje. Este reconocimiento abre nuevas posibilidades de explicación y de intervención. La Ley de Ordenación General del Sistema Educativo (LOGSE) promovió en España un modelo de escuela en donde profesores, alumnos, padres y otras entidades sociales pudiesen desarrollar su propia trayectoria como comunidad escolar. Una comunidad de personas interesadas por transformar y mejorar su propia práctica educativa, capaces de transformar la política educativa y construir un currículo que dé respuestas educativas debidamente contextualizadas según los alumnos y sus entornos socioculturales. La LOE ${ }^{[1]}$ recoge este mismo pensamiento entre sus principios educativos. En el Título II se aborda concretamente este aspecto: la adecuada respuesta a los alumnos se concibe a partir del principio de inclusión, contempla la atención a la diversidad como principio y no como una medida que corresponde a las necesidades de unos pocos. Aboga por un modelo de escuela transmisora y a la vez renovadora de la cultura, regida por los principios de inclusión y equidad, cuyo objetivo es la cohesión social.

Educar en la diversidad implica como señalan Bayot, del Rincón y Hernández Pina
(2002) pensar en una escuela para todos, con unas posibilidades sociales no excluyentes para nadie y en responder a las necesidades de cada alumno de la forma más conveniente para que avance en su crecimiento personal lo máximo posible partiendo de lo que es y de dónde se encuentra.

Una reflexión y valoración compartida de la realidad de su entorno es lo que lleva a responder de forma eficaz a preguntas como: ¿qué educación queremos?, ¿quién la decide?, ¿qué papel corresponde a los centros, profesores y comunidades educativas en cuanto a su diseño, ejecución y evaluación?, ¿qué significa atender a la diversidad del alumno?. Entrar en este proceso de debate es lo enriquecedor para el centro, de no ser así, la elaboración de los distintos niveles de concreción curricular puede convertirse en un mero trámite administrativo sin valor para transformar la práctica cotidiana del mismo. Para ello es necesario recoger gran cantidad de información relacionada con los conocimientos previos de los alumnos, intereses, expectativas, historia educativa, situación social y cultural de sus familias, expectativas de los padres, cultura grupal y las características y oportunidades que ofrece el entorno.

Estamos ante una concepción social en la que es preciso transformar el proceso educativo para dar una respuesta de calidad a la diversidad y lograr aulas eficaces donde la inclusión sea el principio rector. La inclusión de formas de ser, maneras de aprender, de entender la cultura y de todo lo que representa un tratamiento individualizado de la educación. Casanova (1998) señala que además de los planteamientos curriculares generales, se hace necesario un modelo de organización escolar que permita y favorezca la puesta en práctica de las opciones educativas y curriculares decididas.

González y Sabaté (1991) y González (1993) establecieron una serie de principios en los que debe basarse la organización de 
Muñoz Cantero, J.M., Casar Domínguez, L. S. y Abalde Paz, E. (2007). El “contexto" y las "metas y objetivos" como elementos clave en la calidad de la atención a la diversidad en centros no universitarios.

RELIEVE, v. 13, n. 2, p. 235-261. http://www.uv.es/RELIEVE/v13n2/RELIEVEv13n2 6.htm

un centro educativo y que actualmente siguen en vigor

- Flexibilidad: debe de tener una estructura estable y sólida pero sin ser rígida, que acepte las propuestas fruto de la evaluación o nuevas iniciativas e integrarlas en la vida del centro si se juzgan apropiadas. - Apertura y creatividad: en el sentido de impulsar una cultura de colaboración entre todos sus integrantes posibilitando la renovación continua y que fomente el espíritu crítico. Es decir, una escuela inclusiva en la que todos tengan cabida.

- Operatividad funcional: una organización fundamentada en un punto de vista realista y que tenga una proyección práctica.

- Participación y consenso: una organización democrática en la que todos los estamentos y miembros de la comunidad educativa participen activamente en la toma de decisiones.

- Formación: debe de contemplarse desde el proyecto organizativo la formación permanente y el trabajo en equipo de los docentes para hacer frente a la atención a la diversidad.

Los investigadores han intentado identificar aquellas características de las instituciones educativas que, supuestamente, las capacitarían para dar respuesta a la diversidad. En este sentido Reynolds (1985) establece una serie de principios para lograr un desarrollo organizativo adecuado en los centros para lograr una atención de calidad.

- El desarrollo organizativo deberá centrarse más en el trabajo colaborativo del centro que en el trabajo de cada docente individual en clase.

- Dirigido hacia la solución de problemas.

- Se intentará un cambio en la conducta real antes que pretender cambiar las actitudes de algunos miembros del grupo.

- El desarrollo organizativo no puede ser impuesto desde fuera deberá ser responsabilidad del personal de los centros.

- El desarrollo organizativo debe de llevarse a cabo de abajo a arriba antes que de arriba abajo, participando activamente los profesores del centro.

- Se requieren procesos constantes de evaluación de sus efectos y su conocimiento por parte de los implicados, para que se pueda juzgar la efectividad de los cambios.

- El calendario deberá establecerse a largo plazo.

- Se atenderá tanto la organización formal, los procesos escolares como las relaciones existentes entre los miembros de la organización.

Esta aportación de Reynolds no es única. Existen otras que, en esta misma línea, se interesan por determinar las características principales relacionadas con la organización de los centros educativos inclusivas que tiendan a una mayor calidad de la educación (Ramasut y Reynolds, 1993; Rouse y Florian, 1996, etc.).

Gairin (1992, 1998), Jiménez y Vilá (1999) y Zabalza (1998) señalan algunas de las condiciones que pueden facilitar los aspectos organizativos de un proyecto educativo respetuoso con la diversidad de las necesidades del alumnado.

- Capacidad para que el centro educativo, concretamente su profesorado, rompa con el esquema que su actuación se dirige a un "destinatario-imaginario". Esto tiene que ver con la sensibilidad profesional y con la asunción de la comprensividad y la inclusión como valores educativos.

- Calidad y riqueza en los dispositivos, procedimientos y estrategias organizativas. Aunque en este caso, se trata de un tema de técnicas y no de actitudes, es también una cuestión relacionada con la formación.

- Implicación adecuada de los sistemas de apoyo habilitados en el centro escolar o fuera de él (profesores de apoyo, departamentos de orientación, servicios psicopedagógicos, médicos, etc.) para dar soporte al trabajo de cada profesor. No obstante es conveniente evitar una hipertrofia excesi- 
Muñoz Cantero, J.M., Casar Domínguez, L. S. y Abalde Paz, E. (2007). El “contexto" y las "metas y objetivos" como elementos clave en la calidad de la atención a la diversidad en centros no universitarios.

RELIEVE, v. 13, n. 2, p. 235-261. http://www.uv.es/RELIEVE/v13n2/RELIEVEv13n2 6.htm

va de estos recursos especiales ya que en último término, lo que se consigue es volver a acciones y actitudes segregadoras y potenciadoras de desigualdad.

- Capacidad de la propia estructura curricular planteada en cada centro educativo para permitir la diversificación de la oferta formativa de acuerdo a las características del alumnado.

- Superación del compromiso individual en la respuesta a la diversidad, imponiéndose la implicación del centro en su totalidad (principios explícitos en el PEC) y la creación de aquellos mecanismos institucionales (modificaciones organizativas, procesos de formación de centros, adquisición de recursos, desarrollo de iniciativas a propósito de la atención a la diversidad, etc.) que incorporen la diversidad como algo inherente y normal a la vida del centro.

- Predisposición y capacidad del centro y sus integrantes de saber vivir en un entorno de cierta ambigüedad e incertidumbre, en situaciones en las que coexistan posiciones y actuaciones de distinta índole y compromisos en lo que concierne a la respuesta educativa a la diversidad.

- Reconocimiento del centro educativo como una institución marcada notablemente por lo que se piensa y se hace fuera de ella. La escuela no es un contexto libre y la tendencia a la homogeneización presente en todos los ámbitos de la vida repercute, inevitablemente, en la forma en que percibe, analiza y valora el trabajo realizado en ella.

La educación en la actualidad se encuentra en un proceso de reestructuración. Estos cambios exigen pensar en la necesidad de reformas institucionales que adapten sus metas y objetivos a las nuevas necesidades sociales, pero sin perder de vista la finalidad última del sistema, su eficacia (Cabrera, Bethencourt, Álvarez Pérez, y González Afonso, 2006). La educación es una actividad propositiva, intencional y, en consecuencia, la determinación de si se logran, y en qué grado, las metas es un elemento constitutivo de la propia acción. No sería razonable desentenderse de los efectos que justifican la actividad (Orden, Asensio, Carballo, Fernández Díaz, Fuentes, García Ramos y Guardia, 1997).

\section{PLANTEAMIENTO DE LA INVESTIGACIÓN}

El procedimiento metodológico que hemos seguido será el siguiente: planteamiento de la investigación, objetivos, variables, muestra, instrumentos, análisis de datos y conclusiones (Buendía, 1993, Ríos de Deus, 2003; Rassen, 2005; Diniz, 2006).

Nos preocupa la calidad con relación a la atención a la diversidad en nuestros centros. Ésto nos conduce a preguntarnos cuáles serán los indicadores que permitan analizar la calidad de los centros en relación a la atención a la diversidad. En palabras de González López (2003) el gran reto para la educación es la búsqueda de la calidad, relacionada con la necesidad de dar a los estudiantes una formación integral. Por esta razón es fundamental la explicitación de todos aquellos elementos que ayuden a establecer un sistema de estimación de la calidad de la institución educativa.

Para llevar a cabo la investigación nos planteamos en primer lugar elaborar un instrumento de medida, que permita conocer la opinión de los profesionales directamente implicados en la educación y descubrir cual es su opinión respecto a éste tema. Un instrumento que permita medir de forma independiente distintos aspectos de la calidad en la atención a la diversidad de un centro. Y, por tanto, hacer una evaluación parcial de aquellos elementos que nos interesan sin necesidad de obligarnos a realizar una evaluación global.

Creemos en la multidimensionalidad del concepto calidad, en la calidad educativa y 
en la atención a la diversidad desde la óptica de la inclusión. Es por ello que el instrumento que hemos elaborado recoge los aspectos más significativos de la legislación, de otros autores en sus investigaciones y de los profesionales de la educación (profesores, inspectores de educación primaria y secundaria).

Se ha procedido a la elaboración del cuestionario, discusión y recogida de la información para su posterior análisis y posteriormente, se han analizado los datos y se han sacado las conclusiones. En el presente trabajo nos centramos en analizar los resultados relativos a la importancia que los profesores dan al "Contexto" en el que está el centro inmerso y a las "Metas y objetivos" que éstos definen como fruto de sus proyectos curriculares y, posteriormente, analizamos el grado de cumplimiento en los centros.

\subsection{Objetivos}

- A partir de lo dicho anteriormente y teniendo presente que nuestra obligación última es la de mejorar la calidad en la atención a la diversidad, a través de planes de mejora, que los centros pueden llevar a cabo partiendo de una evaluación previa, nos planteamos entre otros los siguiente objetivo:

- La construcción de un instrumento fiable y válido que nos proporcione información de forma global y de forma independiente sobre la importancia de determinados elementos que analizan la atención a la diversidad y su calidad.

- Analizar el grado de importancia y cumplimiento en los centros educativos de ca- da uno de los cuestionarios ("Contexto y Metas y Objetivos") en base al modelo diseñado para evaluar la calidad en la atención a la diversidad.

- Detectar los puntos fuertes y débiles y, por consiguiente, establecer propuestas de mejora en los centros educativos.

\subsection{Variables}

Trabajaremos con los elementos identificativos del "Contexto" del centro educativo y las "Metas y Objetivos" que un centro debe de tener en cuenta para una atención a la diversidad de calidad. Estos elementos forman parte de las variables "Contexto" y "Proceso" respectivamente del modelo CIPP, como veremos más adelante.

\subsection{Instrumento de medida}

Para la elaboración del instrumento general que permitiese una evaluación global de un centro respecto a la atención a la diversidad se confeccionaron seis instrumentos diferentes en torno a un eje común: "Calidad y atención a la diversidad". Los diferentes cuestionarios siguieron las siguientes fases (tabla $\mathrm{n}^{\circ}$ 1): a) recopilación bibliográfica, b) determinación de las dimensiones de partida, c) elaboración de un banco de 250 ítems procedentes de los indicadores de calidad y el marco legislativo, d) elaboración del cuestionario final de 172 ítems con la colaboración de expertos. En esta fase se dispusieron los ítems en las dimensiones en función de su contenido, depuración y redacción de los ítems y e) redacción de los cuestionarios finales. 
Muñoz Cantero, J.M., Casar Domínguez, L. S. y Abalde Paz, E. (2007). El “contexto" y las "metas y objetivos" como elementos clave en la calidad de la atención a la diversidad en centros no universitarios.

RELIEVE, v. 13, n. 2, p. 235-261. http://www.uv.es/RELIEVE/v13n2/RELIEVEv13n2 6.htm

Tabla nº : Fases de elaboración del intrumento "Calidad y Atención a la Diversidad"[1]

\begin{tabular}{|l|l|l|}
\hline FASE 1 & Búsqueda y recopilación bibliográfica. & $\begin{array}{l}\text { Análisis de lecturas de artículos, libros, legisla- } \\
\text { ción, ... }\end{array}$ \\
\hline FASE 2 & $\begin{array}{l}\text { Análisis de la estructura multidimensional del } \\
\text { objeto a estudio. }\end{array}$ & $\begin{array}{l}\text { Determinación del número de } \\
\text { dimensiones de partida } \\
\text { (4 dimensiones) }\end{array}$ \\
\hline FASE 3 & $\begin{array}{l}\text { Revisión y elaboración del banco inicial } \\
\text { de Îtems }\end{array}$ & $\begin{array}{l}\text { Banco de 250 items procedentes de } \\
\text { análisis de indicadores de calidad } \\
\text { y ámbito marco legislativo. }\end{array}$ \\
\hline FASE 4 & $\begin{array}{l}\text { Mediante la colaboración de expertos: } \\
\text { Ubicación de ítems, en función de } \\
\text { su contenido en las dimensiones } \\
\text { predeterminadas. } \\
\text { Depuración y redacción de los ítems. }\end{array}$ & $\begin{array}{l}\text { Elaboración del cuestionario final 172 } \\
\text { Ítems. Distribución en 6 cuestionarios } \\
\text { parciales. }\end{array}$ \\
\hline FASE 5 & Diseño de los cuestionarios del estudio. & $\begin{array}{l}\text { Escala cuantitativa del 1 al 5, } \\
\text { que representan cinco alternativas } \\
\text { continuas. } \\
\text { Instrucciones y forma de aplicación. }\end{array}$ \\
\hline
\end{tabular}

Una de las características esenciales de la calidad es su carácter multidimensional o multifacético; por tanto los diferentes instrumentos se confeccionaron atendiendo a esa multidimensionalidad, partiendo del modelo CIPP de Stuflebeam (1987). No tanto por su filosofía orientada a la toma de decisiones, eficientista, cuantitativista y objetivista sino por su estructura (contexto, entrada, proceso y producto), que se perfila como un modelo idóneo para nuestros objetivos y, nos permite distribuir las dimensiones y factores que consideramos importantes de cara a establecer una serie de indicadores que de- terminen una atención a la diversidad de calidad.

En la gráfica $n^{\circ} 1$ se pueden ver los diferentes cuestionarios (individuales para permitir la evaluación parcial en un centro) de que se compone el instrumento siguiendo las directrices del modelo CIPP de Stufflebeam (1987) (Context, Input -instalaciones y recursos materiales y humanos-, Process metas y objetivos y programa de formación-, Product -resultados obtenidos-). Siendo uno de los más empleados en trabajos evaluativos (Expósito, Olmedo y Fernández Cano, 2004; De Miguel et al., 1994; de Deus, 2003; Rassen, 2005 y Diniz, 2006). 
Muñoz Cantero, J.M., Casar Domínguez, L. S. y Abalde Paz, E. (2007). El “contexto" y las "metas y objetivos" como elementos clave en la calidad de la atención a la diversidad en centros no universitarios.

RELIEVE, v. 13, n. 2, p. 235-261. http://www.uv.es/RELIEVE/v13n2/RELIEVEv13n2 6.htm

Gráfica $n^{\circ} 1$ : instrumento para una inclusión de calidad en centros educativos

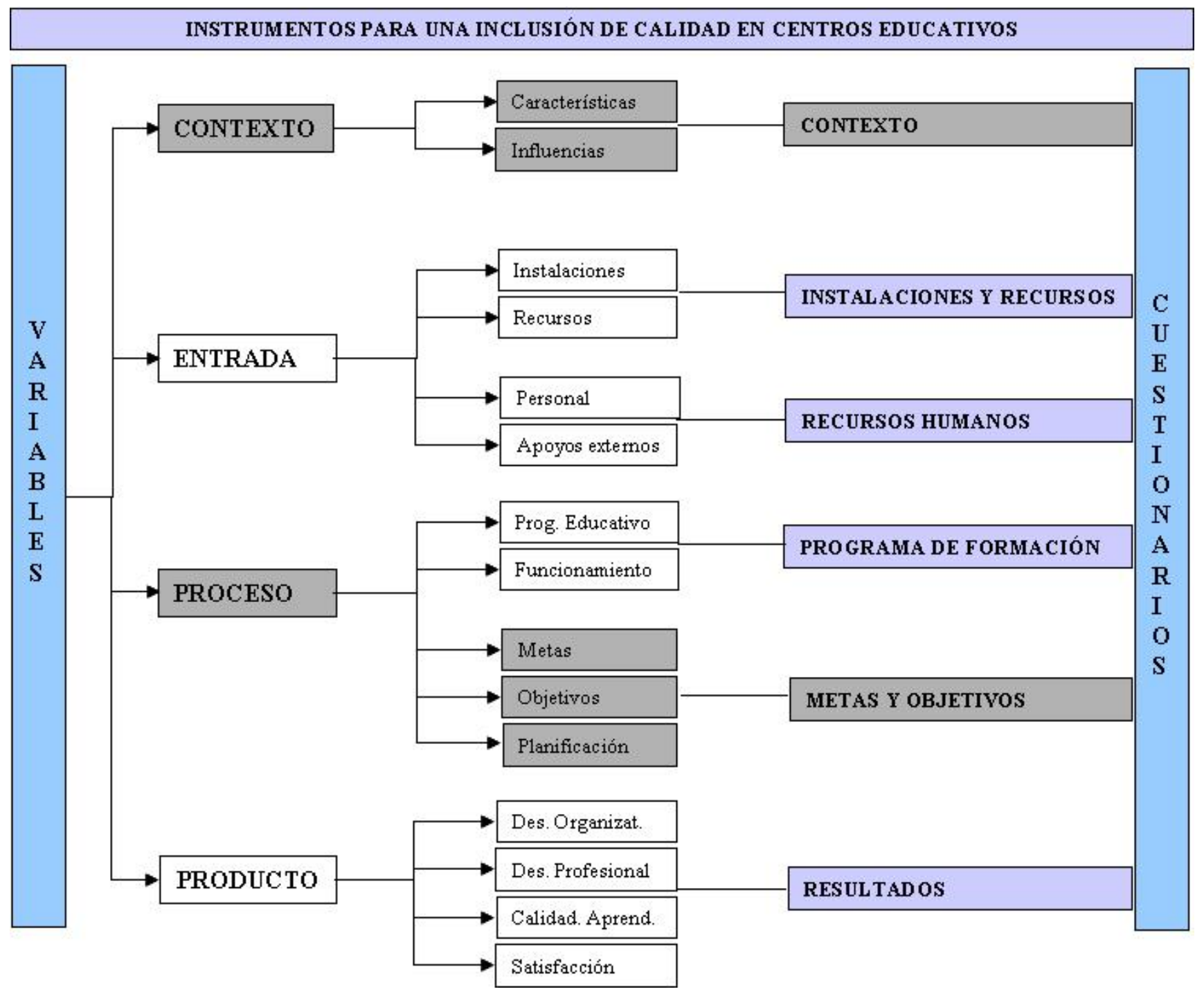

Los seis cuestionarios de evaluación responden a las dimensiones señaladas y la fiabilidad de todos ellos oscilan entre 0,8153 del cuestionario "contexto" y 0,9793 del "programa de formación" (tabla $\mathrm{n}^{\circ} 2$ ). Cada uno de ellos recoge información en cuanto al grado de importancia que los profesores dan a cada uno de los ítems que lo conforman y el nivel de cumplimento en el centro. En los seis cuestionarios se condensan los factores de calidad de los centros educativos en relación a la atención a la diversidad. Además permite que el profesor exprese los puntos fuertes, débiles y propuestas de mejora que, con respecto a los aspectos analizada, considere oportuno. Este artículo se centra en dos dimensiones ya señaladas: "Contexto" y "Metas y objetivos". Toda información adicional sobre estos instrumentos se puede consultar en Casar (2007). 
Muñoz Cantero, J.M., Casar Domínguez, L. S. y Abalde Paz, E. (2007). El “contexto" y las "metas y objetivos" como elementos clave en la calidad de la atención a la diversidad en centros no universitarios.

RELIEVE, v. 13, n. 2, p. 235-261. http://www.uv.es/RELIEVE/v13n2/RELIEVEv13n2 6.htm

Tabla $n^{\circ}$ 2. "Cuestionarios del instrumento "Calidad y Atención a la Diversidad"

\begin{tabular}{|c|c|c|}
\hline CUESTIONARIO & FACTORES INICIALES & $\begin{array}{c}\mathbf{n}^{0} \\
\text { items }\end{array}$ \\
\hline \multirow[t]{2}{*}{ CONTEXTO } & Factor I:"Contexto en el que se encuentra el centro" & 6 \\
\hline & Factor II: "Relaciones externas" & 4 \\
\hline \multirow{2}{*}{$\begin{array}{l}\text { METAS Y } \\
\text { OBJETIVOS }\end{array}$} & Factor I: "Definición de metas y objetivos" & 8 \\
\hline & Factor II: "Planificación" & 5 \\
\hline \multirow{15}{*}{$\begin{array}{l}\text { PROGRAMA } \\
\text { DE FORMACIÓN }\end{array}$} & Factor I: "Organización de la enseñanza" & 5 \\
\hline & Factor II: "El PEC" & 5 \\
\hline & $\begin{array}{l}\text { Factor III:"El PCC: aspectos que el equipo docente debe de tener en cuen- } \\
\text { ta" }\end{array}$ & 11 \\
\hline & Factor IV: "El PCC: organización de espacios" & 4 \\
\hline & Factor V: "Análisis del currículo oficial del PCC" & 7 \\
\hline & Factor VI: "Decisiones metodológicas" & 10 \\
\hline & Factor VII: "Selección de grupos y adscripción a ciclos" & 7 \\
\hline & Factor VIII: "Criterios de promoción" & 3 \\
\hline & $\begin{array}{l}\text { Factor IX: "Las programaciones específicas curriculares: programación de } \\
\text { aula" }\end{array}$ & 3 \\
\hline & $\begin{array}{l}\text { Factor X: "Las programaciones específicas curriculares: Adaptaciones } \\
\text { curriculares" }\end{array}$ & 5 \\
\hline & $\begin{array}{l}\text { Factor XI: "Las programaciones específicas curriculares: refuerzo educati- } \\
\text { vo" }\end{array}$ & 3 \\
\hline & $\begin{array}{l}\text { Factor XII: "Las programaciones específicas organizativas: agrupamientos } \\
\text { específicos" }\end{array}$ & 2 \\
\hline & $\begin{array}{l}\text { Factor XIII: "Las programaciones específicas organizativas: medidas de } \\
\text { apoyo y promoción" }\end{array}$ & 2 \\
\hline & $\begin{array}{l}\text { Factor XIV: "Las programaciones específicas organizativas: programas de } \\
\text { diversificación curricular" }\end{array}$ & 3 \\
\hline & $\begin{array}{l}\text { Factor XV: "Las programaciones específicas organizativas: programas de } \\
\text { garantía social" }\end{array}$ & 3 \\
\hline \multirow{6}{*}{$\begin{array}{l}\text { RECURSOS } \\
\text { HUMANOS }\end{array}$} & Factor I: "Aspectos generales" & 7 \\
\hline & Factor II: "Profesorado" & 6 \\
\hline & Factor III: "Tutor" & 12 \\
\hline & Factor IV: "Profesor de apoyo" & 6 \\
\hline & Factor V: "Departamento de orientación" & 10 \\
\hline & Factor VI: "Equipos de orientación específicos" & 7 \\
\hline $\begin{array}{l}\text { INSTALACIONES } \\
\text { RECURSOS }\end{array}$ & Factor I: "Instalaciones y recursos" & 6 \\
\hline \multirow{2}{*}{ RESULTADOS } & Factor I: "Elementos" & 16 \\
\hline & Factor II: "Estilo de enseñanza" & 6 \\
\hline
\end{tabular}

\subsection{Selección y composición de la muestra.}

La muestra seleccionada necesitaba ser amplia con el fin de garantizar la participación de grupos de diversas condiciones institucionales y diferentes etapas de enseñanza, que se correspondiesen con distintos modos de entender su cometido profesional. La muestra es el resultado de elegir al azar 120 centros de la provincia de la Coruña. El nú- cleo de muestra final que han contestado a los cuestionarios está formado por 1562 profesores de Primaria, Secundaria y Bachillerato pertenecientes a 82 centros educativos $(24$ de centros privados y 58 de centros públicos). Tenemos una mortalidad experimental de 65 sujetos que no han contestado numerosos ítems y que no hemos introducido para los análisis de fiabilidad. La distribución de la muestra es la siguiente: 
Muñoz Cantero, J.M., Casar Domínguez, L. S. y Abalde Paz, E. (2007). El “contexto" y las "metas y objetivos" como elementos clave en la calidad de la atención a la diversidad en centros no universitarios.

RELIEVE, v. 13, n. 2, p. 235-261. http://www.uv.es/RELIEVE/v13n2/RELIEVEv13n2 6.htm

- En función del tipo de centro educativo tenemos 384 sujetos $(24,8 \%)$ pertenecientes a colegios privados (principalmente de núcleos urbanos) y 1162 de centros públi$\cos (75,2 \%)$.

- Teniendo en cuenta los años de docencia, nos encontramos que de 16-30 años de docencia se encuentra el $42 \%$ aproximadamente de los sujetos, aunque entre 0 15 años nos encontramos el 48\%. La mortandad para los análisis de contraste es del $8,6 \%$.

- Incluimos, en la variable desempeño profesional, tres categorías. Aunque la categoría más amplia es la de profesores (70,9\%), hemos considerado, lógicamente, a profesores especialistas (de audición y lenguaje, pedagogía terapéutica, orientadores,... que estén trabajándolo o hayan trabajo con este rol) y profesores que estén o hayan estado como cargos directivos $(9,5 \%)$. Tenemos una mortandad del 9,6\% de los sujetos que no han contestado a esta pregunta del cuestionario.

- En cuanto a la variable etapa decir que casi el $45 \%$ de los sujetos son profesores de secundaria y el $41 \%$ de primaria; siendo la categoría que tiene menos sujetos la de bachillerato. Tenemos una mortalidad de 120 sujetos que no han contestado a este elemento.

\section{Análisis de datos}

El cuestionario "Contexto" contiene 10 ítems distribuidos en dos grupos para recoger los aspectos a valorar en el entorno del centro y las relaciones del centro con el medio. En el cuestionario "Metas y Objetivo se introdujeron 13 ítems estructurados en dos factores: definición de metas y objetivos y planificación. (tabla $n^{\circ} 3$ ). En la definición de metas y objetivos han de tenerse en cuenta aspectos como los recursos humanos, la estructura organizativa, la tipología de centro en relación con su contexto, las infraestructuras, la viabilidad de los objetivos propuestos, que son el marco de referencia de sus decisiones y actividades y la planificación como herramienta que incrementa la eficacia de un centro en la consecución de los objetivos. Además, en cada apartado, se valoran también los principales puntos débiles, fuertes y propuestas de mejora que propondrían los profesores para favorecer una mayor calidad en la atención a la diversidad, con ello se ofrece la posibilidad de dar una respuesta más cualitativa.

Tabla $n^{\circ}$ 3: Factores iniciales del cuestionario “Contexto" y "Metas y objetivos".

\begin{tabular}{|l|l|c|}
\hline \multirow{2}{*}{$\begin{array}{l}\text { CONTEXTO } \\
\text { (10 ítems) }\end{array}$} & $\begin{array}{l}\text { Contexto en el que se } \\
\text { encuentra el centro }\end{array}$ & 6 \\
\cline { 2 - 3 } & Relaciones externas & 4 \\
\hline $\begin{array}{l}\text { METAS Y } \\
\text { OBJETIVOS } \\
\text { (13 ítems) }\end{array}$ & $\begin{array}{l}\text { Definición de metas y } \\
\text { objetivos }\end{array}$ & 8 \\
\cline { 2 - 3 } & Planificación & 5 \\
\hline
\end{tabular}

Para realizar el análisis tomaremos ambos bloques, que los entendemos como el marco de referencia de las decisiones y actividades que los centros deben tomar en relación con su contexto a la hora de establecer su política de actuación. Realizado el análisis de la fiabilidad y de validez de constructo procederemos a efectuar el análisis descriptivo del grado de importancia y el nivel de cumplimiento.

- Importancia: se solicita que valoren cada elemento del cuestionario en función de la importancia que le dan para obtener una atención de calidad.

- Cumplimiento: se solicita que valoren cada elemento en función del grado de cumplimiento que se da en sus centros.

De cara al análisis de los datos hemos de decir que si el ítem es considerado importante (puntuaciones medias por encima de 3,66 ) $\mathrm{y}$ tiene un nivel de cumplimiento por encima del mismo valor señalado lo podremos considerar como puntos fuertes del centro educativo. Por el contrario los valores que se 
Muñoz Cantero, J.M., Casar Domínguez, L. S. y Abalde Paz, E. (2007). El “contexto" y las "metas y objetivos" como elementos clave en la calidad de la atención a la diversidad en centros no universitarios.

RELIEVE, v. 13, n. 2, p. 235-261. http://www.uv.es/RELIEVE/v13n2/RELIEVEv13n2 6.htm

encuentran entre 1 y 2,33, los interpretaremos como puntos débiles. Todos los elementos que se encuentran entre 2,34 y 3,65 que marcan buen nivel de cumplimiento y son considerados importantes, no por ello debemos considerarlos como puntos fuertes o factores de calidad del centro educativo.

\subsection{Análisis de la fiabilidad}

Se ha obtenido para el "Contexto" un coeficiente de fiabilidad del cuestionario de 10 ítems ( $\mathrm{n}=1512$ sujetos) de 0,8153 lo que nos indica un índice de fiabilidad aceptable. Para las "Metas y Objetivos" el coeficiente de fiabilidad del cuestionario fue para $\operatorname{los} 13$ ítems de 0,9197, un índice de fiabilidad alto. El comportamiento de los ítems ha sido, en general bueno, ya que ninguno de ellos afectaba al coeficiente de fiabilidad de forma que disminuyese su valor tal como podemos observar en la tabla $\mathrm{n}^{\mathrm{o}} 4$ y tabla $\mathrm{n}^{\mathrm{o}} 5$. referida al análisis de la fiabilidad en la que en la última columna los valores reflejan su incidencia en este índice al disminuir si se elimina el ítem.

Tabla $n^{\circ} 4$ : Fiabilidad. Contexto

\begin{tabular}{|c|c|c|c|c|}
\hline & $\begin{array}{l}\text { Media } \\
\text { de la } \\
\text { escala si } \\
\text { el item } \\
\text { es eli- } \\
\text { minado }\end{array}$ & $\begin{array}{l}\text { Varianza } \\
\text { de la escala } \\
\text { si elimina- } \\
\text { mos el item }\end{array}$ & $\begin{array}{c}\text { Correlación } \\
\text { Item-Total } \\
\text { corregida }\end{array}$ & $\begin{array}{c}\text { Alpha } \\
\text { si el } \\
\text { item } \\
\text { es } \\
\text { elimi- } \\
\text { nado }\end{array}$ \\
\hline $\begin{array}{l}\text { El contexto socioeconómico y cultural en el que se encuentra } \\
\text { situado el centro }\end{array}$ & 35,7407 & 24,9955 & ,3616 &, 8143 \\
\hline Las características del alumnado & 35,1844 & 24,4900 & 3459 & 8014 \\
\hline $\begin{array}{l}\text { La posibilidad de intercambio (social, cultural,...) con el } \\
\text { entorno }\end{array}$ & 35,7593 & 24,0253 & ,4107 &, 7925 \\
\hline $\begin{array}{l}\text { Las posibilidades de intercambio de información y trabajo } \\
\text { conjunto con las familias }\end{array}$ & 35,2650 & 24,3781 & ,3607 & ,7956 \\
\hline $\begin{array}{l}\text { El grado de autonomía que tiene el centro con relación a la } \\
\text { atención a la diversidad }\end{array}$ & 35,4949 & 25,2815 & ,2810 &, 8053 \\
\hline $\begin{array}{l}\text { La posibilidad de inserción profesional del alumnado con } \\
\text { necesidades educativas especiales (NEE) }\end{array}$ & 35,4462 & 23,3824 & ,3120 &, 8001 \\
\hline La relación cooperativa con otros centros & 35,9270 & 24,2842 & , 4479 & ,7947 \\
\hline $\begin{array}{l}\text { Las relación regulares del profesorado con otros/-as profeso- } \\
\text { res/-as y especialistas }\end{array}$ & 35,4392 & 23,7327 & ,4483 & ,7911 \\
\hline $\begin{array}{l}\text { La existencia de programas de cooperación con otras institu- } \\
\text { ciones educativas, sociales,... }\end{array}$ & 35,6440 & 24,6240 & ,3955 & ,7978 \\
\hline $\begin{array}{l}\text { Intercambios de información y trabajo conjunto con Servicios } \\
\text { Sociales y Servicios Hospitalarios }\end{array}$ & 35,6050 & 23,7125 & 4001 & ,7949 \\
\hline
\end{tabular}


Muñoz Cantero, J.M., Casar Domínguez, L. S. y Abalde Paz, E. (2007). El “contexto" y las "metas y objetivos" como elementos clave en la calidad de la atención a la diversidad en centros no universitarios.

RELIEVE, v. 13, n. 2, p. 235-261. http://www.uv.es/RELIEVE/v13n2/RELIEVEv13n2 6.htm

Tabla $n^{\circ} 5$ - Análisis de fiabilidad - metas y objetivos

1. Tomando como referencia el proyecto educativo de centro, el grado de consenso en los objetivos del centro con relación a la atención a la diversidad

2. Tomando como referencia el proyecto educativo de centro, el grado de conocimiento por parte de la comunidad educativa (claustro de profesores/-as, consejo escolar,...)

3. Para la definición de las metas y objetivos, tener en cuenta los recursos humanos

4. Para la definición de las metas y objetivos, tener en cuenta a estructura organizativa

5. Para la definición de las metas y objetivos, tener en cuenta factores como la tipología del centro en relación con su contexto

6. Para la definición de las metas y objetivos, tener en cuenta las infraestructuras

7. Para la definición de las metas y objetivos, tener en cuenta la viabilidad de los objetivos propuestos

8. Establecer procedimientos para el seguimiento y revisión de los objetivos

9. Existencia de una programación que permita conseguir los objetivos y metas con relación a la atención a la diversidad (programación de medidas de atención a la diversidad)

10. Para la elaboración de la planificación de los objetivos, tener en cuenta los puntos fuertes y débiles en relación a la atención a la diversidad

11. Para la elaboración de la planificación de los objetivos, tener en cuenta las oportunidades del contorno social del centro

12. Para la elaboración de la planificación de los objetivos, tener en cuenta el nivel de coordinación entre los planes estratégicos (proyecto curricular de centro -PCC-, proyecto educativo de centro -PEC-, programaciónes,...) y los de atención a la diversidad

13. Para la elaboración de la planificación de los objetivos, tener en cuenta el grado de conocimiento y acuerdo que existe entre los miembros de la comunidad educativa sobre los objetivos y las acciones prioritarias que se proponen para mejorar la calidad de la atención a la diversidad

\begin{tabular}{|c|c|c|c|}
$\begin{array}{c}\text { Media de } \\
\text { la escala } \\
\text { si el item } \\
\text { es elimi- } \\
\text { nado }\end{array}$ & $\begin{array}{c}\text { Varianza } \\
\text { de la esca- } \\
\text { la si eli- } \\
\text { minamos } \\
\text { el item }\end{array}$ & $\begin{array}{c}\text { Correlación } \\
\text { Item-Total } \\
\text { corregida }\end{array}$ & $\begin{array}{c}\text { Alpha si } \\
\text { el item es } \\
\text { elimina- } \\
\text { do }\end{array}$ \\
\hline 49,3598 & 48,1651 &, 5869 &, 9161 \\
\hline 49,3150 & 47,8687 &, 6104 &, 9153 \\
\hline 49,3143 & 46,8858 &, 7141 &, 9115 \\
\hline 49,4533 & 46,1493 &, 7304 &, 9107 \\
\hline 49,5819 & 46,1389 &, 6832 &, 9125 \\
\hline 49,3976 & 45,6759 &, 7661 &, 9092 \\
\hline 49,4981 & 46,1797 &, 6778 &, 9127 \\
\hline 49,1255 & 48,4865 &, 6239 &, 9150 \\
\hline 49,5896 & 46,6816 &, 6494 &, 9138 \\
\hline 49,8560 & 46,9241 &, 5710 &, 9174 \\
\hline 4,5570 & 46,9503 &, 6329 &, 9145 \\
\hline
\end{tabular}

mos los pasos siguientes: en primer lugar calcularemos el valor de KMO para los ítems referenciados, en segundo lugar realizaremos el análisis factorial de componentes principales con rotación varimax y por últimos calcu- 
Muñoz Cantero, J.M., Casar Domínguez, L. S. y Abalde Paz, E. (2007). El “contexto" y las "metas y objetivos" como elementos clave en la calidad de la atención a la diversidad en centros no universitarios.

RELIEVE, v. 13, n. 2, p. 235-261. http://www.uv.es/RELIEVE/v13n2/RELIEVEv13n2 6.htm

laremos la varianza explicada por el factor si fuese el caso.

\section{“Contexto"}

Presenta un valor prácticamente de cero del determinante de la matriz de correlaciones $(0,055)$ lo que nos indica la existencia de intercorrelaciones muy altas entre las variables. A partir de los valores (muy altos) obtenidos en el test de esfericidad de Barlett (tabla $\mathrm{n}^{\mathrm{o}}$ 6) con un Chi Cuadrado de 4525,444 y $\mathrm{p}=0,000$, rechazamos la hipótesis nula de que la matriz de correlaciones es una matriz identidad, indicando la existencia de intercorrelaciones significativas entre los ítems para el cuestionario.

El índice KMO (tabla ${ }^{\circ}$ 6) es bueno según el baremo de interpretación $(0,802)$ lo que nos indica que podemos continuar con el análisis factorial.

Tabla ${ }^{\circ}$ 6: KMO y prueba de Bartlett. Contexto

\begin{tabular}{|l|l|c|}
\hline $\begin{array}{l}\text { Medida de adecuación muestral de Kaiser- } \\
\text { Meyer-Olkin. }\end{array}$ &, 802 \\
$\begin{array}{l}\text { Prueba de esfericidad de } \\
\text { Bartlett }\end{array}$ & $\begin{array}{l}\text { Chi-cuadrado } \\
\text { aproximado }\end{array}$ & 4525,444 \\
& gl & 45 \\
& Sig. &, 000 \\
\hline
\end{tabular}

El procedimiento seguido en la obtención de factores es del de componentes principales. La primera de las columnas de la tabla $\mathrm{n}^{\circ}$ 7 muestra los ítems que han entrado en análi- sis y, en la segunda, las comunalidades. Vemos que el comportamiento de los ítems es desigual desde valores que oscilan entre 0,473 del ítem 12 a 0,889 del ítem 29.

Tabla n ${ }^{\circ}$ 7: Método de extracción: Análisis de Componentes principales. Comunalidades - Contexto

\begin{tabular}{|c|c|c|}
\hline & Inicial & Extracción \\
\hline 1. El contexto socioeconómico y cultural en el que se encuentra situado el centro & 1,000 & ,772 \\
\hline 2. Las características del alumnado & 1,000 & ,502 \\
\hline 3. La posibilidad de intercambio (social, cultural,...) con el entorno & 1,000 & ,531 \\
\hline 4. Las posibilidades de intercambio de información y trabajo conjunto con las familias & 1,000 & ,615 \\
\hline 5. El grado de autonomía que tiene el centro con relación a la atención a la diversidad & 1,000 & 653 \\
\hline $\begin{array}{l}\text { 6. La posibilidad de inserción profesional del alumnado con necesidades educativas espe- } \\
\text { ciales (NEE) }\end{array}$ & 1,000 & ,517 \\
\hline 7. La relación cooperativa con otros centros & 1,000 & ,704 \\
\hline 8. Las relaciones habituales del profesorado con otros/-as profesores/-as y especialistas & 1,000 & ,619 \\
\hline $\begin{array}{l}\text { 9. La existencia de programas de cooperación con otras instituciones educativas, socia- } \\
\text { les,... }\end{array}$ & 1,000 & ,635 \\
\hline $\begin{array}{l}\text { 10. Intercambios de información y trabajo conjunto con Servicios Sociales y Servicios } \\
\text { Hospitalarios }\end{array}$ & 1,000 & 605 \\
\hline
\end{tabular}

En las tablas siguientes se presenta el análisis factorial con rotación varimax. Se obtiene una matriz de 3 componentes en el cues- tionario una vez rotada la matriz con procedimiento varimax. 
Muñoz Cantero, J.M., Casar Domínguez, L. S. y Abalde Paz, E. (2007). El “contexto" y las "metas y objetivos" como elementos clave en la calidad de la atención a la diversidad en centros no universitarios.

RELIEVE, v. 13, n. 2, p. 235-261. http://www.uv.es/RELIEVE/v13n2/RELIEVEv13n2_6.htm

Tabla $n^{\circ}$ 8: Método de extracción: análisis de componentes principales a 3 componentes extraídos

\begin{tabular}{|l|c|c|c|}
\hline & \multicolumn{2}{|c|}{ COMPONENTES } \\
\hline $\begin{array}{l}\text { 9. La existencia de programas de cooperación con otras instituciones educativas, socia- } \\
\text { les,... }\end{array}$ & 1 & 2 & 3 \\
\hline 7. La relación cooperativa con otros centros &, 772 & \\
\hline $\begin{array}{l}\text { 8. Las relaciones regulares del profesorado con otros/-as profesores/-as y especialistas } \\
\text { 10. Intercambios de información y trabajo conjunto con Servicios Sociales y Servicios } \\
\text { Hospitalarios }\end{array}$ &, 743 &, 729 \\
\hline $\begin{array}{l}\text { 5. Grado de autonomía que tiene el centro con relación a la atención a la diversidad } \\
\text { 4. Las posibilidades de intercambio de información y trabajo conjunto con las familias }\end{array}$ & & \\
\hline $\begin{array}{l}\text { 6. La posibilidad de inserción profesional del alumnado con necesidades educativas } \\
\text { especiales (NEE) }\end{array}$ & &, 786 \\
\hline $\begin{array}{l}\text { 1. El contexto socioeconómico y cultural en el que se encuentra situado el centro } \\
\text { 3. La posibilidad de intercambio (social, cultural,...) con el entorno }\end{array}$ &, 612 & \\
\hline 2. Las características del alumnado &, 346 \\
\hline
\end{tabular}

Como se puede observar en la tabla de la varianza total explicada (tabla $n^{\circ} 9$ ), estos tres factores explican el $61,526 \%$ de la va- rianza total, explicando el primero de ellos el $24,991 \%$.

Tabla $n^{\circ} 9$ : Varianza total explicada. Método de extracción: Análisis de Componentes principales. Dimensión contexto

\begin{tabular}{|c|c|c|c|c|c|c|c|c|c|}
\hline \multirow[t]{2}{*}{ Componente } & \multicolumn{3}{|c|}{ Autovalores iniciales } & \multicolumn{3}{|c|}{$\begin{array}{c}\text { Sumas de las saturaciones } \\
\text { al cuadrado de la extrac- } \\
\text { ción }\end{array}$} & \multicolumn{3}{|c|}{$\begin{array}{l}\text { Suma de las saturaciones al } \\
\text { cuadrado de la rotación }\end{array}$} \\
\hline & Total & $\begin{array}{l}\% \text { de } \\
\text { la va- } \\
\text { rianza }\end{array}$ & $\begin{array}{l}\% \text { acumula- } \\
\text { do }\end{array}$ & Total & $\begin{array}{l}\% \text { de la } \\
\text { varian- } \\
\text { za }\end{array}$ & $\begin{array}{c}\% \\
\text { acumu- } \\
\text { lado }\end{array}$ & Total & $\begin{array}{l}\% \text { de } \\
\text { la va- } \\
\text { rianza }\end{array}$ & $\begin{array}{l}\% \text { acumula- } \\
\text { do }\end{array}$ \\
\hline 1 & 3,822 & 38,220 & 38,220 & 3,822 & 38,220 & 38,220 & 2,499 & 24,991 & 24,991 \\
\hline 2 & 1,317 & 13,165 & 51,386 & 1,317 & 13,165 & 51,386 & 1,941 & 19,407 & 44,398 \\
\hline 3 & 1,014 & 10,140 & 61,526 & 1,014 & 10,140 & 61,526 & 1,713 & 17,128 & 61,526 \\
\hline 4 & ,893 & 8,933 & 70,459 & & & & & & \\
\hline 5 & 695 & 6,951 & 77,410 & & & & & & \\
\hline 6 & ,571 & 5,705 & 83,115 & & & & & & \\
\hline 7 &, 514 & 5,141 & 88,256 & & & & & & \\
\hline 8 & ,447 & 4,467 & 92,722 & & & & & & \\
\hline 9 & ,391 & 3,910 & 96,633 & & & & & & \\
\hline 10 & ,337 & 3,367 & 100,000 & & & & & & \\
\hline
\end{tabular}

Interpretación de los factores del Cuestionario

- Factor I: Relaciones externas al centro
Este factor se refiere a aspectos referidos a las "relaciones externas del centro" (ítems 7 al 10). Estos ítems se mantienen según la estructura planteada en el modelo inicial. 
Muñoz Cantero, J.M., Casar Domínguez, L. S. y Abalde Paz, E. (2007). El “contexto" y las "metas y objetivos" como elementos clave en la calidad de la atención a la diversidad en centros no universitarios.

RELIEVE, v. 13, n. 2, p. 235-261. http://www.uv.es/RELIEVE/v13n2/RELIEVEv13n2 6.htm

Tabla $n^{\circ}$ 10: Componentes del factor I (relaciones externas al centro)

\begin{tabular}{|l|c|c|c|}
\hline & $\mathbf{3}$ & $\mathbf{2}$ & $\mathbf{3}$ \\
\hline $\begin{array}{l}\text { 9. La existencia de programas de cooperación con otras } \\
\text { instituciones educativas, sociales,... }\end{array}$ &, 775 & & \\
\hline $\begin{array}{l}\text { 7. La relación cooperativa con otros centros } \\
\text { 8. Las relaciones habituales del profesorado con otros/-as } \\
\text { profesores/-as y especialistas }\end{array}$ &, 772 & &, 327 \\
\hline $\begin{array}{l}\text { 10. Intercambios de información y trabajo conjunto con } \\
\text { Servicios Sociales y Servicios Hospitalarios }\end{array}$ &, 729 & \\
\hline FIABILIDAD ,7907 & & \\
\hline
\end{tabular}

\section{- Factor II: Relación Centro-entorno}

Este segundo factor recoge aquellos ítems que hacen referencia a la relación del centro con el contorno. El ítem $6^{\circ}$ comparte peso con el tercero de los factores, aunque mantiene su mayor peso en el $2^{\circ}$.

Tabla $n^{\circ}$ 11: Componentes del factor II (centro-entorno)

\begin{tabular}{|l|c|c|c|}
\hline & \multicolumn{3}{|c|}{ Componentes } \\
\hline $\begin{array}{l}\text { 5. El grado de autonomía que tiene el centro con relación a la } \\
\text { atención a la diversidad }\end{array}$ & & $\mathbf{2}$ & $\mathbf{3}$ \\
\hline $\begin{array}{l}\text { 4. Las posibilidades de intercambio de información y trabajo } \\
\text { conjunto con las familias }\end{array}$ &, 727 & \\
$\begin{array}{l}\text { 6. La posibilidad de inserción profesional del alumnado con } \\
\text { necesidades educativas especiales (NEE) }\end{array}$ &, 612 &, 346 \\
\hline FIABILIDAD , 8032
\end{tabular}

- Factor III: Contexto socio-económico y cultural

Este tercer factor lo forman los tres primeros ítems del cuestionario y están referidos a aspectos globales del entorno. Dos de ellos $\left(\mathrm{el} 2^{\circ}\right.$ y $3^{\circ}$ ) comparten carga factorial (con el $2^{\mathrm{o}}$ y $1^{\mathrm{o}}$ factor respectivamente); aunque $\mathrm{su}$ peso lo tienen en este factor.

Tabla $n^{\circ} 12$. : Componentes del factor III (contexto socioeconómico y cultural)

\begin{tabular}{|l|c|c|c|}
\hline & \multicolumn{3}{|c|}{ Componentes } \\
\hline $\begin{array}{l}\text { 1. El contexto socioeconómico y cultural en el que } \\
\text { se encontra situado elcentro }\end{array}$ & & $\mathbf{2}$ & $\mathbf{3}$ \\
\hline 2. Las características del alumnado & &, 876 \\
\hline $\begin{array}{l}\text { 3. La posibilidad de intercambio (social, cultural,...) } \\
\text { con el entorno }\end{array}$ &, 341 &, 550 \\
\hline FIABILIDAD ,6032 & &, 579 \\
\hline
\end{tabular}

En términos generales vemos que los ítems del segundo y tercer factor comparten peso entre ellos lo que muestra la relación existente entre ellos. 
Muñoz Cantero, J.M., Casar Domínguez, L. S. y Abalde Paz, E. (2007). El “contexto" y las "metas y objetivos" como elementos clave en la calidad de la atención a la diversidad en centros no universitarios.

RELIEVE, v. 13, n. 2, p. 235-261. http://www.uv.es/RELIEVE/v13n2/RELIEVEv13n2 6.htm

\section{“Metas y Objetivos"}

Presenta un valor prácticamente de 0 del determinante de la matriz de correlaciones $(0,001)$ que nos indica la existencia de intercorrelaciones muy altas entre las variables.

A partir de los valores muy altos obtenidos en el test de esfericidad de Barlett (tabla $\mathrm{n}^{\circ}$ 24) con un Chi Cuadrado de 11419,502 y p
$=, 000$, rechazamos la hipótesis nula de que la matriz de correlaciones es una matriz identi$\mathrm{dad}$, indicando la existencia de intercorrelaciones significativas entre los ítems para el cuestionario.

El índice KMO (tabla $n^{\circ} 13$ ) es excelente según el baremo de interpretación $(0,915)$ lo que nos indica que podemos continuar con el análisis factorial.

Tabla $n^{\circ}$ 13: KMO y prueba de Bartlett. Dimensión Metas y objetivos

\begin{tabular}{|l|l|c|c|}
\hline \multicolumn{3}{|l|}{ Medida de adecuación muestral de Kaiser-Meyer-Olkin. } &, 915 \\
$\begin{array}{l}\text { Prueba de esfericidad de } \\
\text { Bartlett }\end{array}$ & Chi-cuadrado aproximado & 11419,502 \\
& gl & 78 \\
& Sig. &, 000 \\
\hline
\end{tabular}

El procedimiento seguido en la obtención de factores es del de componentes principales. La primera de las columnas de la tabla $\mathrm{n}^{\circ}$ 14 muestra los ítems que han entrado en aná- lisis $\mathrm{y}$, en la segunda, las comunalidades. Vemos que el comportamiento de los ítems es desigual desde valores que oscilan entre 0,590 del ítem 8 a 0,771 del ítem 11

Tabla n ${ }^{\circ}$ 14: Método de extracción: Análisis de Componentes principales. Comunalidades.

Dimensión Metas y objetivos

1. Tomando como referencia el proyecto educativo de centro, el grado de consenso en los objetivos del centro con relación a la atención a la diversidad

2. Tomando como referencia el proyecto educativo de centro, el grado de conocimiento por parte de la comunidad educativa (claustro de profesores/-as, consello escolar,...)

3. Para la definición de las metas y objetivos, tener en cuenta los recursos humanos

4. Para la definición de las metas y objetivos, tener en cuenta la estructura organizativa

5. Para la definición de las metas y objetivos, tener en cuenta la tipología del centro en relación con su contexto

6. Para la definición de las metas y objetivos, tener en cuenta las infraestructuras

7. Para la definición de las metas y objetivos, tener en cuenta la viabilidad de los objetivos propuestosstos

8. Establecer procedimientos para el seguimento y revisión de los objetivos

9. Existencia de una programación que permita conseguir los objetivos y metas con relación a la atención a la diversidad (programación de medidas de atención a la diversidad)

10. Para la elaboración da planificación de los objetivos, tener en cuenta los puntos fuertes y débiles con relación a la atención a la diversidad

11. Para la elaboración de la planificación de los objetivos, tener en cuenta las oportunidades del contorno social del centro

12. Para a elaboración de la planificación de los objetivos, tener en cuenta el nivel de coordinación entre los planes estratégicos (proyecto curricular de centro -PCC-, proyecto educativo de centro -PEC-, programaciones,...) y los de atención a la diversidad

13. Para a elaboración de la planificación de los objetivos, tener en cuenta el grado de conocimiento y acuerdo que existe entre los miembros de la comunidad educativa sobre los objetivos y las acciones prioritarias que se proponen para mejorar la calidad de la atención a la diversidad

\begin{tabular}{|c|c|}
\hline Inicial & Extracción \\
\hline 1,000 &, 768 \\
\hline 1,000 &, 741 \\
\hline 1,000 &, 744 \\
\hline 1,000 &, 768 \\
\hline 1,000 &, 609 \\
\hline 1,000 &, 632 \\
\hline 1,000 &, 711 \\
\hline 1,000 &, 590 \\
\hline 1,000 &, 617 \\
\hline 1,000 &, 689 \\
\hline 1,000 &, 771 \\
\hline 1,000 &, 561 \\
\hline 1,000 &, 627 \\
\hline
\end{tabular}


Muñoz Cantero, J.M., Casar Domínguez, L. S. y Abalde Paz, E. (2007). El “contexto" y las "metas y objetivos" como elementos clave en la calidad de la atención a la diversidad en centros no universitarios.

RELIEVE, v. 13, n. 2, p. 235-261. http://www.uv.es/RELIEVE/v13n2/RELIEVEv13n2 6.htm

En las tablas siguientes se presenta el análisis factorial con rotación varimax. Se obtiene una matriz de 3 componentes en el cuestionario. Hemos de observar que aunque se reflejan tres componentes el primero de ellos reúne a todos los ítems con una carga factorial más grande. Es por esta razón por la que sólo vamos a considerar un factor para análisis e interpretaciones posteriores.

Tabla $n^{\circ}$ 15: Método de extracción: Análisis de componentes principales a 3 componentes extraídos

\begin{tabular}{|c|c|c|c|}
\hline & \multicolumn{3}{|c|}{ COMPONENTES } \\
\hline & 1 & 2 & 3 \\
\hline $\begin{array}{l}\text { 7. Para la definición de las metas y objetivos, tener en cuenta la viabilidad de los } \\
\text { objetivos propuestos }\end{array}$ & ,816 & & \\
\hline $\begin{array}{l}\text { 4. Para la definición de las metas y objetivos, tener en cuenta la estructura organi- } \\
\text { zativa }\end{array}$ & ,788 &,- 336 & \\
\hline $\begin{array}{l}\text { 3. Para la definición de las metas y objetivos, tener en cuenta los recursos huma- } \\
\text { nos }\end{array}$ & ,773 & & \\
\hline 8. Establecer procedimientos para el seguimiento y revisión de los objetivos & ,740 & & \\
\hline $\begin{array}{l}\text { 5. Para la definición de las metas y objetivos, tener en cuenta factores como la } \\
\text { tipología del centro en relación con su contexto }\end{array}$ & ,739 & & \\
\hline 6. Para la definición de las metas y objetivos, tener en cuenta las infraestructuras & ,719 &,- 308 & \\
\hline $\begin{array}{l}\text { 10. Para la elaboración da planificación dos objetivos, tener en cuenta os puntos } \\
\text { fuertes y débiles con relación a la atención a la diversidad }\end{array}$ & ,702 & & ,424 \\
\hline $\begin{array}{l}\text { 12. Para la elaboración de la planificación de los objetivos, tener en cuenta el } \\
\text { nivel de coordinación entre los planes estratégicos (proyecto curricular de centro } \\
\text {-PCC-, proyecto educativo de centro -PEC-, programaciones,...) y los de atención } \\
\text { a la diversidad }\end{array}$ & ,690 & & \\
\hline $\begin{array}{l}\text { 13. Para la elaboración de la planificación de los objetivos, tener en cuenta el } \\
\text { conocimiento y acuerdo que existe entre los miembros de la comunidad educativa } \\
\text { sobre los objetivos y las acciones prioritarias que se proponen para mejorar la } \\
\text { calidad da atención a la diversidad }\end{array}$ & ,688 & ,354 & \\
\hline $\begin{array}{l}\text { 9. Existencia de una programación que permita conseguir los objetivos y metas } \\
\text { con relación a la atención a la diversidad (programación de medidas de atención a } \\
\text { la diversidad) }\end{array}$ & ,685 & ,367 & \\
\hline $\begin{array}{l}\text { 2. Tomando como referencia el proyecto educativo de centro, el grado de cono- } \\
\text { cimiento por parte da comunidad educativa (claustro de profesores/-as, consejo } \\
\text { escolar,...) }\end{array}$ & 676 & ,371 &,- 383 \\
\hline $\begin{array}{l}\text { 1. Tomando como referencia el proyecto educativo de centro, el grado de consen- } \\
\text { so nos objetivos del centro con relación a la atención a la diversidad }\end{array}$ & ,653 & ,470 &,- 348 \\
\hline $\begin{array}{l}\text { 11. Para la elaboración de la planificación de los objetivos, tener en cuenta las } \\
\text { oportunidades del entorno social del centro }\end{array}$ & ,627 & & 600 \\
\hline
\end{tabular}

Como se puede observar en la tabla de la varianza total explicada (tabla $\mathrm{n}^{\mathrm{o}} 7$ ), estos tres factores explican el $67,917 \%$ de la va- rianza total, explicando el primero de ellos el $29,917 \%$. 
Muñoz Cantero, J.M., Casar Domínguez, L. S. y Abalde Paz, E. (2007). El “contexto" y las "metas y objetivos" como elementos clave en la calidad de la atención a la diversidad en centros no universitarios.

RELIEVE, v. 13, n. 2, p. 235-261. http://www.uv.es/RELIEVE/v13n2/RELIEVEv13n2 6.htm

Tabla n 16: Varianza total explicada. Método de extracción: Análisis de Componentes principales.

Dimensión Metas y objetivos

\begin{tabular}{|c|c|c|c|c|c|c|c|c|c|}
\hline \multirow[t]{2}{*}{ Componente } & \multicolumn{3}{|c|}{ Autovalores iniciales } & \multicolumn{3}{|c|}{$\begin{array}{l}\text { Sumas de las saturaciones al } \\
\text { cuadrado de la extracción }\end{array}$} & \multicolumn{3}{|c|}{$\begin{array}{l}\text { Suma de las saturaciones al } \\
\text { cuadrado de la rotación }\end{array}$} \\
\hline & Total & $\begin{array}{l}\% \text { de la } \\
\text { varianza }\end{array}$ & $\begin{array}{l}\% \text { acu- } \\
\text { mulado }\end{array}$ & Total & $\begin{array}{c}\% \text { de la } \\
\text { varianza }\end{array}$ & $\begin{array}{l}\text { \% acu- } \\
\text { mulado }\end{array}$ & Total & $\begin{array}{c}\% \text { de la } \\
\text { varianza }\end{array}$ & $\begin{array}{l}\text { \% acu- } \\
\text { mulado }\end{array}$ \\
\hline 1 & 6,683 & 51,408 & 51,408 & 6,683 & 51,408 & 51,408 & 3,773 & 29,025 & 29,025 \\
\hline 2 & 1,078 & 8,293 & 59,701 & 1,078 & 8,293 & 59,701 & 2,588 & 19,908 & 48,932 \\
\hline 3 & 1,068 & 8,216 & 67,917 & 1,068 & 8,216 & 67,917 & 2,468 & 18,985 & 67,917 \\
\hline 4 &, 702 & 5,403 & 73,320 & & & & & & \\
\hline 5 & ,625 & 4,806 & 78,125 & & & & & & \\
\hline 6 &, 503 & 3,866 & 81,992 & & & & & & \\
\hline 7 &, 453 & 3,488 & 85,479 & & & & & & \\
\hline 8 &, 426 & 3,277 & 88,756 & & & & & & \\
\hline 9 &, 346 & 2,659 & 91,415 & & & & & & \\
\hline 10 & ,327 & 2,513 & 93,928 & & & & & & \\
\hline 11 & ,306 & 2,357 & 96,285 & & & & & & \\
\hline 12 &, 274 & 2,109 & 98,394 & & & & & & \\
\hline 13 & ,209 & 1,606 & 100,000 & & & & & & \\
\hline
\end{tabular}

\subsection{Análisis descriptivo: identificación de puntos fuertes y débiles}

Por término medio podemos ver en la tabla siguiente (tabla $\left.\mathrm{n}^{\mathrm{o}} 17\right)$ referida a los estadísticos descriptivos referentes al "Contexto" que los indicadores han sido bien valorados, lo que nos indica que estos ítems son importantes para dar una atención de calidad tomando como referencia la atención a la diversidad. La desviación típica es aceptable siendo la opinión menos homogénea en el ítem referido "La posibilidad de inserción profesional del alumnado con necesidades educativas especiales (NEE)" en el contexto en que se encuadran los centros con un valor de 1,034 .

Por lo que hace referencia al grado de “cumplimiento" (tabla $\mathrm{n}^{\circ} 17$ ) vemos que, en primer lugar las valoraciones bajan; lo que nos indican que, aunque los consideran importantes, el grado en que estos elementos son tenidos en cuenta de cara a la atención a la diversidad es menor pues todos los valores son más bajos; encontramos valores por debajo de 3 en los ítems 6, 7, 9 y 10; aunque también es en estos ítems donde encontra- mos una mayor desviación lo que nos indicaría que no hay tanta homogeneidad en su opinión.

En términos generales podríamos decir que:

- Todos los elementos señalados tienen un nivel de cumplimiento medio (valores entre 2,34 y 3,65 ) y dado que no tenemos elementos por debajo de 2,34 , podemos decir que existe un grado de cumplimiento medio en los centros y debemos considerarlos como puntos a mejorar y susceptibles de establecer propuestas de mejora en la orientación de cada uno de ellos de cara a mejorar el contexto en el que se encuentran los centros, especialmente:

- Establecer mecanismos de inserción laboral de alumnos con N.E.E.

- Establecer mecanismos de cooperación e intercambio con otros centros educativos y otras instituciones sociales, hospitalarias, etc.

- Solamente el ítem 2 obtiene valores por encima de 3,65 con lo que, además de ser uno de los ítems más valorados en su grado de importancia también es uno de los tenidos 
Muñoz Cantero, J.M., Casar Domínguez, L. S. y Abalde Paz, E. (2007). El “contexto" y las "metas y objetivos" como elementos clave en la calidad de la atención a la diversidad en centros no universitarios.

RELIEVE, v. 13, n. 2, p. 235-261. http://www.uv.es/RELIEVE/v13n2/RELIEVEv13n2 6.htm

más en cuenta de cara a mejorar la calidad de la atención a la diversidad. Se señala como punto fuerte:
- Atención a las características del alumnado.

Tabla $n^{\circ}$ 17: Estadísticos descriptivos - contexto

\begin{tabular}{|c|c|c|c|c|c|c|}
\hline & \multicolumn{3}{|c|}{ IMPORTANCIA } & \multicolumn{3}{|c|}{ CUMPLIMIENTO } \\
\hline & $\mathrm{N}$ & Media & $\begin{array}{l}\text { Desv. } \\
\text { típ. }\end{array}$ & $\mathrm{N}$ & Media & $\begin{array}{l}\text { Desv. } \\
\text { típ. }\end{array}$ \\
\hline $\begin{array}{l}\text { 1. El contexto socioeconómico y cultural en el que se } \\
\text { encuentra situado el centro }\end{array}$ & 1562 & 3,76 & ,959 & 1561 & 3,37 & ,924 \\
\hline 2. Las características del alumnado & 1562 & 4,32 & ,883 & 1561 & 3,70 & ,910 \\
\hline $\begin{array}{l}\text { 3. La posibilidad de intercambio (social, cultural,...) } \\
\text { con el entorno }\end{array}$ & 1562 & 3,74 & ,851 & 1561 & 3,08 & ,948 \\
\hline $\begin{array}{l}\text { 4. Las posibilidades de intercambio de información y } \\
\text { trabajo conjunto con las familias }\end{array}$ & 1562 & 4,24 & ,828 & 1562 & 3,19 & 1,101 \\
\hline $\begin{array}{l}\text { 5. El grado de autonomía que tiene el centro con } \\
\text { relación a la atención a la diversidad }\end{array}$ & 1562 & 4,01 & ,800 & 1562 & 3,31 & ,994 \\
\hline $\begin{array}{l}\text { 6. La posibilidad de inserción profesional del alum- } \\
\text { nado con necesidades educativas especiales (NEE) }\end{array}$ & 1562 & 4,05 & 1,034 & 1562 & 2,83 & 947 \\
\hline 7. La relación cooperativa con otros centros & 1562 & 3,57 & ,833 & 1562 & 2,80 & 1,001 \\
\hline $\begin{array}{l}\text { 8. Las relaciones habituales del profesorado con } \\
\text { otros/as profesores/as y especialistas }\end{array}$ & 1562 & 4,06 & ,883 & 1562 & 3,08 & 1,086 \\
\hline $\begin{array}{l}\text { 9. La existencia de programas de cooperación con } \\
\text { ootras instituciones educativas, sociales, } \ldots\end{array}$ & 1562 & 3,86 & ,814 & 1562 & 2,77 & 1,024 \\
\hline $\begin{array}{l}\text { 10. Intercambios de información y trabajo conjunto } \\
\text { con Servicios Sociales y Servicios Hospitalarios }\end{array}$ & 1562 & 3,90 & 931 & 1562 & 2,97 & 1,107 \\
\hline
\end{tabular}

Realizaremos a continuación la valoración de las opiniones manifestadas de forma abierta por los profesores respecto al objeto de valoración, en función de las valoraciones que hicieron en el cuestionario. Para llevar a cabo este análisis se les pidió que señalaran los puntos fuertes de su centro, los puntos débiles y las propuestas de mejora para lo- grar una atención a la diversidad de calidad en sus respectivos centros educativos. Tras la recopilación de estos datos se procedió a agrupar las respuestas mayoritarias (tabla $\mathrm{n}^{\circ}$ 18). Si observamos la tabla $n^{\circ} 17$ vemos que existe una gran relación entre lo manifestado cuantitativamente y lo descrito cualitativamente. 
Muñoz Cantero, J.M., Casar Domínguez, L. S. y Abalde Paz, E. (2007). El “contexto" y las "metas y objetivos" como elementos clave en la calidad de la atención a la diversidad en centros no universitarios.

RELIEVE, v. 13, n. 2, p. 235-261. http://www.uv.es/RELIEVE/v13n2/RELIEVEv13n2 6.htm

Tabla $n^{\circ}$ 18: Opiniones del profesorado en cuanto al contexto

\begin{tabular}{|c|c|c|c|}
\hline FACTOR & PUNTOS FUERTES & PUNTOS DÉBILES & $\begin{array}{l}\text { PROPUESTAS DE ME- } \\
\text { JORA }\end{array}$ \\
\hline $\begin{array}{l}\text { Factor I: "Relaciones } \\
\text { externas del centro" }\end{array}$ & $\begin{array}{l}\text { - Relación con otros centros } \\
\text { - Trabajo conjunto con los } \\
\text { servicios sociales } \\
\text { - Cooperación con otras } \\
\text { instituciones }\end{array}$ & $\begin{array}{l}\text { - Falta de cooperación con } \\
\text { otros centros } \\
\text { - Poco apoyo de las institu- } \\
\text { ciones } \\
\text { - Escasez del tiempo de dedi- } \\
\text { cación de la orientadora } \\
\text { - Poca relación del profeso- } \\
\text { rado con otros especialistas } \\
\text { - Falta de dotación económi- } \\
\text { ca por parte de la administra- } \\
\text { ción }\end{array}$ & $\begin{array}{l}\text { - Mejorar la cooperación con } \\
\text { otros centros } \\
\text { - Mayor horario de orienta- } \\
\text { ción en el centro } \\
\text { - Medios económicos. } \\
\text { - Proyectos de cooperación } \\
\text { concretos. } \\
\text { - Cooperación y apoyo de las } \\
\text { instituciones } \\
\text { - Recursos humanos y cursos } \\
\text { de formación }\end{array}$ \\
\hline $\begin{array}{l}\text { Factor II: } \\
\text { "Relación } \\
\text { entorno" }\end{array}$ & $\begin{array}{l}\text { - Relación con los pa- } \\
\text { dres/madres } \\
\text { - Intercambio con el entorno }\end{array}$ & $\begin{array}{l}\text { - Ausencia de una relación } \\
\text { fluida con las familias } \\
\text { - Falta de intercambio con el } \\
\text { entorno } \\
\text { - Grado de autonomía bajo } \\
\text { - Poca posibilidad de inser- } \\
\text { ción profesional }\end{array}$ & $\begin{array}{l}\text { - Mantener una relación más } \\
\text { fluida y coordinada con las } \\
\text { familias y APAs } \\
\text { - Posibilidades de intercam- } \\
\text { bio con el entorno } \\
\text { - Inserción profesional }\end{array}$ \\
\hline $\begin{array}{l}\text { Factor III: "Contexto } \\
\text { socio-económico y } \\
\text { cultural" }\end{array}$ & $\begin{array}{l}\text { - Tener en cuenta las caracte- } \\
\text { rísticas de los alumnos }\end{array}$ & $\begin{array}{l}\text { - Masificación de Minorías } \\
\text { étnicas en los grupos } \\
\text { - Contexto en que se encuen- } \\
\text { tra el centro }\end{array}$ & $\begin{array}{l}\text { - No concentrar toda la po- } \\
\text { blación marginal en un mis- } \\
\text { mo centro }\end{array}$ \\
\hline
\end{tabular}

En lo que respecta a las "Metas y objetivos" podemos ver en la tabla $\mathrm{n}^{\circ} 19$, referida a los estadísticos descriptivos, que los ítems son mejor valorados ${ }^{[2]}$ en lo referente a la importancia que éstos tienen de cara a una mejor calidad en atención a la diversidad, siendo menores los valores obtenidos en cuanto al nivel de cumplimiento en los centros. La desviación típica muestra, así mismo, que la opinión es más homogénea en cuanto al grado de importancia que le conceden, frente al nivel de cumplimiento, en donde encontramos valores de 1 o muy próximos lo que nos indica una mayor heterogeneidad de opinión.

El ítem menos valorado es el ítem 11 que hace referencia a si en la planificación de objetivos se tiene en cuenta el contexto social, lo que nos muestra que no es un indica- dor considerado como importante y, por consiguiente, su grado de cumplimiento es bajo.

Todos los demás valores se encuentran o muy próximos a 4 (ítem 5) o por encima (todos los demás) en cuanto a la valoración que se hace respecto a la importancia de estos ítems y, por encima de 3 en lo que hace referencia al cumplimiento. Éstos últimos no sobrepasan de los valores indicados para ser considerados puntos fuertes $(3,65)$ y tampoco tenemos ninguno por debajo de 2,34 para ser considerados como puntos débiles.

Sin embargo, si consideramos que deberían plantearse propuestas de mejora orientadas a conseguir mejores valores en los siguientes indicadores: el consenso y conocimiento de los objetivos por parte de la comunidad escolar, una planificación en consonancia con los recursos, los mecanismos de revisión y la coordinación. 
Muñoz Cantero, J.M., Casar Domínguez, L. S. y Abalde Paz, E. (2007). El “contexto” y las "metas y objetivos" como elementos clave en la calidad de la atención a la diversidad en centros no universitarios.

RELIEVE, v. 13, n. 2, p. 235-261. http://www.uv.es/RELIEVE/v13n2/RELIEVEv13n2 6.htm

Tabla ${ }^{\circ} 19:$ Estadísticos descriptivos Metas y objetivos

\begin{tabular}{|c|c|c|c|c|c|c|}
\hline & \multicolumn{3}{|c|}{ IMPORTANCIA } & \multicolumn{3}{|c|}{ CUMPLIMIENTO } \\
\hline & $\mathrm{N}$ & Media & $\begin{array}{l}\text { Desv. } \\
\text { típ. }\end{array}$ & $\mathrm{N}$ & Media & $\begin{array}{l}\text { Desv. } \\
\text { Típ. }\end{array}$ \\
\hline $\begin{array}{l}\text { 1. Tomando como referencia el proyecto educativo de centro, el gra- } \\
\text { do de consenso con los objetivos del centro en relación a la atención } \\
\text { a la diversidad }\end{array}$ & 1562 & 4,24 & ,736 & 1562 & 3,28 & ,877 \\
\hline $\begin{array}{l}\text { 2. Tomando como referencia el proyecto educativo de centro, el gra- } \\
\text { do de conocimiento por parte de la comunidad educativa (claustro de } \\
\text { profesores/-as, consejo escolar,...) }\end{array}$ & 1562 & 4,28 & ,744 & 1562 & 3,23 & ,965 \\
\hline $\begin{array}{l}\text { 3. Para la definición de las metas y objetivos, tener en cuenta los } \\
\text { recursos humanos }\end{array}$ & 1562 & 4,28 & ,743 & 1562 & 3,36 & ,993 \\
\hline $\begin{array}{l}\text { 4. Para la definición de las metas y objetivos, tener en cuenta la es- } \\
\text { tructura organizativa }\end{array}$ & 1562 & 4,14 & ,798 & 1562 & 3,38 & ,949 \\
\hline $\begin{array}{l}\text { 5. Para la definición de las metas y objetivos, tener en cuenta factores } \\
\text { como la tipología del centro en relación con su contexto }\end{array}$ & 1562 & 3,92 & 846 & 1562 & 3,34 & ,854 \\
\hline $\begin{array}{l}\text { 6. Para la definición de las metas y objetivos, tener en cuenta las } \\
\text { infraestructuras }\end{array}$ & 1562 & 4,02 & 847 & 1562 & 3,28 & ,926 \\
\hline $\begin{array}{l}\text { 7. Para la definición de las metas y objetivos, tener en cuenta la viabi- } \\
\text { lidad de los objetivos propuestos }\end{array}$ & 1562 & 4,20 & ,808 & 1562 & 3,40 & ,856 \\
\hline $\begin{array}{l}\text { 8. Establecer procedimentos para el seguimiento y revisión de los } \\
\text { objetivos }\end{array}$ & 1562 & 4,10 & ,847 & 1562 & 3,19 & ,989 \\
\hline $\begin{array}{l}\text { 9. Existencia de una programación que permita conseguir los objeti- } \\
\text { vos y metas con relación a la atención a la diversidad (programación } \\
\text { de medidas de atención a la diversidad) }\end{array}$ & 1562 & 4,47 & ,665 & 1562 & 3,56 & 1,000 \\
\hline $\begin{array}{l}\text { 10. Para la elaboración da planificación de los objetivos, tener en } \\
\text { cuenta los puntos fuertes y débiles con relación a la atención a la } \\
\text { diversidad }\end{array}$ & 1562 & 4,01 & ,827 & 1562 & 3,29 & ,964 \\
\hline $\begin{array}{l}\text { 11. Para la elaboración de la planificación de los objetivos, tener en } \\
\text { cuenta las oportunidades del contorno social del centro }\end{array}$ & 1562 & 3,74 &, 892 & 1562 & 3,11 & ,924 \\
\hline $\begin{array}{l}\text { 12. Para la elaboración de la planificación de los objetivos, tener en } \\
\text { cuenta el nivel de coordinación entre los planes estratégicos (proyec- } \\
\text { to curricular de centro -PCC-, proyecto educativo de centro -PEC-, } \\
\text { programaciones,...) y los de atención a la diversidad }\end{array}$ & 1562 & 4,04 & 817 & 1562 & 3,27 & 1,004 \\
\hline $\begin{array}{l}\text { 13. Para la elaboración de la planificación de los objetivos, tener en } \\
\text { cuenta el grado de conocimiento y acuerdo que existe entre los } \\
\text { miembros de la comunidad educativa sobre los objetivos y las accio- } \\
\text { nes prioritarias que se proponen para mejorar la calidad de la aten- } \\
\text { ción a la diversidad. }\end{array}$ & 1562 & 4,15 & ,766 & 1562 & 3,22 & 1,022 \\
\hline
\end{tabular}

En la tabla $\mathrm{n}^{\mathrm{o}} 20$ se pueden ver las valoraciones de las opiniones expuestas de forma abierta por los profesores, en el apartado cualitativo del cuestionario, en función de las valoraciones que previamente habían hecho de los ítems del cuestionario.

Este análisis, como señalamos anteriormente, se llevó a cabo pidiendo a los encues- tados que señalaran los puntos fuertes de su centro, los puntos débiles y las propuestas de mejora para lograr una atención a la diversidad de calidad en sus centros educativos. Las respuestas mayoritarias se agruparon en función de cada factor. Nos encontramos nuevamente con una fuerte correspondencia entre lo manifestado cuantitativamente y lo descrito cualitativamente. 
Muñoz Cantero, J.M., Casar Domínguez, L. S. y Abalde Paz, E. (2007). El “contexto" y las "metas y objetivos" como elementos clave en la calidad de la atención a la diversidad en centros no universitarios.

RELIEVE, v. 13, n. 2, p. 235-261. http://www.uv.es/RELIEVE/v13n2/RELIEVEv13n2 6.htm

Tabla $n^{\circ} 20$ : Opiniones del profesorado en cuanto a metas y objetivos.

\begin{tabular}{|c|c|c|c|}
\hline FACTOR & PUNTOS FUERTES & PUNTOS DÉBILES & $\begin{array}{c}\text { PROPUESTAS DE } \\
\text { MEJORA }\end{array}$ \\
\hline $\begin{array}{l}\text { Factor I: } \\
\text { "Metas y } \\
\text { Objetivos" }\end{array}$ & $\begin{array}{l}\text { - Atención personalizada } \\
\text { - Profesorado ilusionado y } \\
\text { sensible ante la atención a la } \\
\text { diversidad. } \\
\text { - Buena coordinación del } \\
\text { equipo docente. } \\
\text { - El equipo de orientación. } \\
\text { - El equipo directivo. } \\
\text { - El trabajo de programa de } \\
\text { refuerzos y adaptaciones. } \\
\text { - Unanimidad de objetivos } \\
\text { para mejorar la atención a la } \\
\text { diversidad teniendo en cuenta } \\
\text { el PEC y PCC. } \\
\text { - Se tiene en cuenta la realidad } \\
\text { del centro para establecer las } \\
\text { metas. } \\
\text { - Se tiene en cuenta la estruc- } \\
\text { tura organizativa. } \\
\text { - Se tienen en cuenta las in- } \\
\text { fraestructuras y los recursos } \\
\text { materiales. } \\
\text { - Buena planificación del } \\
\text { programa de Medidas de } \\
\text { Atención a la Diversidad. }\end{array}$ & $\begin{array}{l}\text { - Provisionalidad o falta de } \\
\text { recursos humanos especializa- } \\
\text { dos (PT, AL, cuidadores). } \\
\text { - Pocas oportunidades de aten- } \\
\text { ción a la diversidad en el con- } \\
\text { torno escolar. } \\
\text { - Bajo conocimiento por parte } \\
\text { del Claustro de las Medidas de } \\
\text { Atención a la Diversidad. } \\
\text { - Falta de interés del profesora- } \\
\text { do. } \\
\text { - Las infraestructuras. } \\
\text { - Escaso seguimiento y revisión } \\
\text { de los objetivos y las planifica- } \\
\text { ciones. } \\
\text { - Masificación de las aulas. } \\
\text { - Falta de medios materiales. } \\
\text { - Poca coordinación. }\end{array}$ & $\begin{array}{l}\text { - Contar con recursos humanos } \\
\text { especializados. } \\
\text { - Objetivos claros, viables y } \\
\text { reflejados en el PEC. } \\
\text { - Conocimiento por parte del } \\
\text { Claustro. } \\
\text { - Adaptar las infraestructuras. } \\
\text { - Seguimiento periódico. } \\
\text { - Recursos materiales. } \\
\text { - Formación del profesorado. } \\
\text { - Canales de comunicación y } \\
\text { coordinación. } \\
\text { - Oportunidades del entorno } \\
\text { social. } \\
\text { - Puesta en práctica de progra- } \\
\text { mas de atención a la diversidad } \\
\text { realistas y consensuados. } \\
\text { - Mayor apoyo de la Adminis- } \\
\text { tración. } \\
\text { - Adaptar el centro a las nece- } \\
\text { sidades. }\end{array}$ \\
\hline
\end{tabular}

\section{4.- Conclusiones}

El cuestionario “Contexto” consta de 10 ítems con un coeficiente de fiabilidad de 0.8153 En el análisis factorial con rotación varimax se obtuvieron tres componentes que representan los factores que se comentan a continuación.

- Factor I: "Relaciones externas al centro": recoge información sobre aspectos de cooperación y trabajo conjunto del centro educativo con otras instituciones u otros centros educativos (ítems del 7 al 10). Nos encontramos que son importantes para el profesorado, pero el grado de cumplimiento no es muy elevando, siendo por tanto aspectos susceptibles de optimizarse. Podemos señalar como punto a mejorar: establecer programas de cooperación e intercambio con otras insti- tuciones (servicios sociales, hospitales, etc.) y otros centros educativos.

- Factor II: "Relaciones centro-entorno": este factor (ítems del 4 al 6) se refiere a aspectos relacionados con el grado de autonomía del centro en cuanto a atención a la diversidad, las relaciones con las familias y las posibilidades de inserción profesional del alumnado con necesidades educativas especiales. Los resultados obtenidos nos indica que sí es importante para el profesorado; sin embargo, el nivel de cumplimiento en los centros no es excelente, por lo que es un factor susceptible de mejora sobre todo en un aspecto que podemos considerarlo como punto débil: la posibilidad de inserción profesional de alumnos con necesidades educativas especiales. 
Muñoz Cantero, J.M., Casar Domínguez, L. S. y Abalde Paz, E. (2007). El “contexto" y las "metas y objetivos" como elementos clave en la calidad de la atención a la diversidad en centros no universitarios.

RELIEVE, v. 13, n. 2, p. 235-261. http://www.uv.es/RELIEVE/v13n2/RELIEVEv13n2 6.htm

- Factor III: “Contexto socio-económico y cultural": los ítems que forman este tercer factor (del 1 al 3) versan sobre el contexto socio-económico y cultural que rodea al centro educativo, las características de los alumnos y las posibilidades de intercambio con el entorno. La observación de los datos que nos proporciona el análisis descriptivo nos indica que nuevamente el grado de importancia para el profesorado es bueno; aunque más bajo que en los otros dos factores de esta dimensión en dos de sus ítems: el $\mathrm{n}^{\mathrm{o}} 1$ que se refiere al contexto sociocultural en el que se encuentra el centro y el ítem $n^{\circ} 3$ que trata sobre la posibilidad de intercambio sobre el entorno. En cuanto al cumplimiento vemos como en los otros casos que es susceptible de mejora, menos el ítem 2 que lo podemos considerar como un punto fuerte del centro: las características del alumnado.

\section{La información de las "Metas y objetivos"} se recoge en 13 ítems. Se obtuvo un coeficiente de fiabilidad de .9197 . Una vez realizado el análisis factorial con rotación varimax se consiguieron tres componentes, aunque todos los ítems se reúnen en el primero de ellos y es en él donde poseen la mayor carga. Por lo que se consideró un único factor.

Este factor se refiere a los elementos a tener en cuenta relacionados con las metas y objetivos de cara a diseñar y planificar los proyectos educativos. Todos los ítems tienen su peso máximo en el primero de los componentes. Ítems como el 1 y 2 comparten peso factorial con los otros dos factores (están referidos al grado de conocimiento y de consenso respecto a los objetivos del proyecto educativo del centro). El 4 y 6 del segundo factor y que comparten con el primero se refieren a estructura e infraestructura del centro a la hora de definir los objetivos. En cuanto a los ítems 10 y 11 del tercer factor hacen referencia a elementos a tener en cuenta para la elaboración de planificación de programaciones (oportunidades entorno social y debilidades y fortalezas del propio centro). La fiabilidad obtenida para este factor es la misma que la calculada anteriormente de 0.9197 .

En este único factor de las "Metas y objetivos" del centro educativo se agrupa la información relativa a: el grado de consenso en los objetivos del centro en relación con la atención a la diversidad, el nivel de conocimiento del proyecto educativo por parte de la comunidad educativa, el seguimiento de los mismos, la existencia de una programación en relación con la consecución de los objetivos relacionados con la atención a la diversidad, y sí por una parte, en la definición de metas y objetivos se tuvieron en cuenta: los recursos humanos, la estructura organizativa, la tipología del centro en relación con su entorno, las infraestructuras, la viabilidad de los objetivos propuestos, y por otra parte, si en la planificación de los objetivos propuestos se tuvieron en cuenta: los puntos fuertes y débiles en relación con la atención a la diversidad, el nivel de coordinación entre los planes estratégicos y los de atención a la diversidad, las oportunidades del entorno social del centro y el grado de conocimiento y consenso para lograr los objetivos y establecer las acciones prioritarias para mejorar la calidad de atención a la diversidad.

El análisis descriptivo de lo datos relativos al factor nos indican que el grado de importancia para el profesorado es alto en todos los ítems, siendo quizá los menos importantes, aunque están dentro de la media de importancia los que se refiere a:

- Para la elaboración de la planificación de los objetivos se tuvo en cuenta las oportunidades del contorno social del centro.

- Para la definición de metas y objetivos se tuvo en cuenta la tipología del centro en relación con su contexto.

En lo que se refiere al cumplimiento en el centro, las medias muestran que todas estas cuestiones son susceptibles de mejora, sin llegar a ser puntos débiles. Dentro de ellos deberían realizarse propuestas de mejora en los siguientes indicadores: 
Muñoz Cantero, J.M., Casar Domínguez, L. S. y Abalde Paz, E. (2007). El “contexto" y las "metas y objetivos" como elementos clave en la calidad de la atención a la diversidad en centros no universitarios.

RELIEVE, v. 13, n. 2, p. 235-261. http://www.uv.es/RELIEVE/v13n2/RELIEVEv13n2 6.htm

- El grado de consenso en los objetivos por parte de la comunidad educativa.

- El conocimiento por parte de la comunidad educativa.

- Tener en cuenta en su definición: los recursos humanos, la estructura organizativa, la infraestructura.

- Establecer mecanismos de revisión.

- Planificar en función de las oportunidades del entorno.

- Grado de coordinación entre los planes estratégicos.

\section{BIBLIOGRAFÍA}

Álvarez Rojo, V.; Rodríguez, A.; García, E.; Gil, J.; López, I.; Romero, S.; Padilla, M.T.; García, J. y Matas, A. (2002). La atención a la diversidad en los centros de educación secundaria: estudio descriptivo de la provincia de Sevilla. Revista de Investigación Educativa, 20(1), 225-247.

Bartolomé, M. (coord.) (1997). Diagnóstico a la escuela multicultural. Barcelona: Cedecs.

Bartolomé, M. (2005). Editorial. Revista de Investigación Educativa, 23(2), 291-292.

Bartolomé, M. y Cabrera, F. (200o). Nuevas tendencias en la evaluación de programas de educación multicultural. Revista de Investigación Educativa, 18(2), 463-48.

Bayot, A., del Rincón, B. y Hernández Pina, F. (2002). Orientación y atención a la diversidad: descripción de programas y acciones en algunos grupos emergentes. RELIEVE, $v$. 8 , n. 1 . Consultado el 10 de enero de 2007 en

http://www.uv.es/RELIEVE/v8n1/RELIEV Ev8n1 2.htm

Buendía, L. (coord.) (1993). Análisis de la investigación educativa. Granada: Universidad de Granada.

Buendía, L. e Hidalgo, V. (2006). La mejora de un centro educativo de carácter multicultural en la ciudad autónoma de Ceuta. Revista de Investigación Educativa, 24(1), 261-281.

Cabrera, Lidia, Bethencourt, José Tomás, Alvarez Pérez, Pedro y González Afonso,
Míriam (2006). El problema del abandono de los estudios universitarios. RELIEVE, $v$. 12, n. 2. Consultado el 10 de enero de 2007 en

http://www.uv.es/RELIEVE/v12n2/RELIE VEv12n2 1.htm

Cardona, J. (2006). Diversidad y educación inclusiva. Madrid: PEARSON

Casanova, M.A. (1992). La evaluación, garantía de calidad del centro educativo. Zaragoza: Edelvives.

Casanova, $M^{\mathrm{a}}$.A. (1995). Manual de Evaluación Educativa. Zaragoza: Edelvives.

Casanova, M.A. (1999). Manual de evaluación educativa. Madrid: Ed. La Muralla,

Casar, S. (2007). Atención a la diversidad. Un estudio de su calidad en centros de la provincia de la Coruña. La Coruña (España): Universidade da Coruña. Tesis Doctoral inédita

De Miguel, M., Madrid, V., Noriega, J. y Rodríguez, B. (1994). Evaluación para la calidad de los Institutos de Educación Secundaria. Madrid: Escuela Española.

De Miguel, M. (1997). La evaluación de los centros educativos. Una aproximación a un enfoque sistémico. Revista de Investigación Educativa. 2, 145-178.

De la Orden, Asensio, I., Carballo, R., Fernández Díaz, J., Fuentes, A., García Ramos, J.M. y Guardia, S. (1997). Desarrollo y validación de un modelo de calidad universitaria como base para su evaluación. RELIEVE, v. 3, n. 1. Consultado el 10 de enero de 2007 en http://www.uv.es/RELIEVE/v3n1/RELIEV Ev3n1 2.htm .

Echeita, G. (2006). Educación para la inclusión educación sin exclusiones. Madrid: Narcea.

Elboj, C. Puigdelllivol, I. Soler, M. y Valls, R. (2002). Comunidades de aprendizaje. Barcelona: Graó.

Escudero, J. M. (1996). La evaluación del proyecto de centro. En N. Illán (Coord): Didáctica y organización en educación especial (pp. 91-108). Malaga: Aljibe

Escudero, J. M. (2001). La educación, puerta de entrada o de exclusión a la sociedad del 
Muñoz Cantero, J.M., Casar Domínguez, L. S. y Abalde Paz, E. (2007). El “contexto" y las "metas y objetivos" como elementos clave en la calidad de la atención a la diversidad en centros no universitarios.

RELIEVE, v. 13, n. 2, p. 235-261. http://www.uv.es/RELIEVE/v13n2/RELIEVEv13n2 6.htm

conocimiento. Murcia: Ponencia presentada en EDUTEC.

Expósito, Jorge, Olmedo, Eva y Fernández Cano, Antonio (2004). Patrones metodológicos en la investigación española sobre evaluación de programas educativos. RELIEVE, v. 10, n. 2. Consultado el $10 \mathrm{de}$ enero de 2007 en http://www.uv.es/RELIEVE/v10n2/RELIE VEv10n2 2.htm.

Flecha, R. (1992). El discurso sobre la educación de las perspectivas postmodernas. En H. A. Giroux y R. Flecha, Igualdad educativa y diferencias culturales (165-196). Barcelona: El Roure Editorial.

Gairín, J. (1991). Planteamientos institucionales de los centros educativos. En Curso de formación para equipos directivos. Madrid: MEC.

Gairín, J. (1992). Las claves culturales de los conflictos en las organizaciones escolares. Ponencia presentada en el II congreso Interuniversitario de Organización Escolar. Sevilla.

Gairín, J. (1998). Estrategias organizativas en la atención a la diversidad. Educar, 2223, 239-267.

Gaírin, J. (1999). Los departamentos y equipos educativos como órganos de desarrollo profesional. Revista de Currículum y Formación del Profesorado, 3(1), 85-114.

González, M.A. y Sabate, J. (1991). La integración de alumnos con necesidades educativas especiales. Madrid: MEC.

González López, Ignacio (2003). Determinación de los elementos que condicionan la calidad de la universidad: aplicación práctica de un análisis factorial. RELIEVE, v. 9, n. 1. Consultado el 10 de enero de 2007 en http://http:/www.uv.es/RELIEVE/v9n1/RE LIEVEv9n1 4.htm

González Jiménez, M.A. (1993). La organización del centro al servicio de la respuesta a la diversidad. Aula de Innovación Educativa, 10, 47-51.

Hernández, M. E. (1996). De la filosofía de la integración a la filosofía de la inclusión. La diversidad en la escuela inclusiva. P. Jurado y otros (Coords.): Las necessitats edu- catives: present i futur. XIII Jornadas de universidad y educación especial (pp. 315320). Bellatera: Universitat Autonoma de Bellaterra.

Jiménez, C. (2001). Educación y diversidad en los más capaces. Hacia su plena integración escolar. Revista de Investigación Educativa, 19(1), 7-39.

Jiménez, F. y Vilá, M. (1999). De educación especial a educación en la diversidad. Malaga: Aljibe .

Marín, N. y Soler, M. (2004). Una comunidad de aprendizaje. Cuadernos de pedagogía, 331, 60-62.

Ley orgánica de ordenación general del sistema educativo (LOGSE).Ley 1/1990 de 3 de octubre. BOE del 4 de octubre.

Ramasut, A. y Reynolds, D. (1993). Developing effective whole school approaches to special educational needs: from school effective theory to school development practice. En R. Slee, Is there a desk with name on it?. The politics of integration (pp. 219240). London: Falmer Press.

Rassen, N. (2005). Evaluación del programa nacional de informática en la educación. Tesis doctoral inedita. La Coruña (España): Universidade da Coruña.

Reynols, M. (1962). A Framework for considering some issues in special education. Exceptional Children, 28, 367-370.

Reynols, M. (1985). Classification of students with handicapas. En E.W. Gordon (Ed.), Review of research in education. Vol II (pp. 63-92). Washington: American Educational Research Association

Rios de Deus, P. Muñoz Cantero, J.M. (2003). Indicadores de evaluación de la investigación en educación superior. Revista gallego-portuguesa de psicología y educación, 10, 93-112.

Rouse, M. Y Florian, L. (1996). Effective inclusive schools: a study in two countries. Cambridge Journal of Inclusive Education, 1 (4), 323-336.

Diniz, A.M. (2006). Construcción y validación de un modelo multidimensional de ajuste de los jóvenes al contexto universitario. Psicothema 18 (2), 249-255. 
Muñoz Cantero, J.M., Casar Domínguez, L. S. y Abalde Paz, E. (2007). El “contexto" y las "metas y objetivos" como elementos clave en la calidad de la atención a la diversidad en centros no universitarios.

RELIEVE, v. 13, n. 2, p. 235-261. http://www.uv.es/RELIEVE/v13n2/RELIEVEv13n2 6.htm

Stainback, S. y Stainback, W. (1990). Support networks for inclusive schooling. Interdependent integrates education. London: Paul Brookes Publishing.

Stainback, S.; Stainback, W. y Moravec, J. (1999). Un currículo para crear aulas inclusivas. En S. Stainback y W. Stainback, $A u-$ las inclusivas (pp. 83-101). Madrid: Narcea. Stufflebeam, D. M. y Shinkfield, A.J. (1987). Evaluación sistémica. Barcelona: Paidós.

Stufflebeam, D. M. y Shinkfield, A.J. (1993). Evaluación sistémica. Guía teórica y práctica. Madrid: Paidos-MEC.

Zabalza, M.A. (1998). Condiciones organizativas de la escuela ante la diversidad. En R. Pérez (Coord.): Educación y diversidad. $X V$ Jornadas Nacionales de Universidad y Educación Especial (pp. 25-38). Oviedo: Servicio Nacional de Publicaciones de la Universidad de Oviedo.

\section{NOTAS}

${ }^{[1]}$ LOE, Ley Orgánica de Educación en España (vigente desde 2006).

${ }^{[2]} \mathrm{Si}$ el ítem es considerado importante (puntuaciones medias por encima de 3,66) y tiene un nivel de cumplimiento por encima del mismo valor lo podremos considerar como puntos fuertes del centro educativo. Por el contrario los valores que se encuentran entre 1 y 2,33, serán puntos débiles o puntos no importantes. Todos los elementos que se encuentran entre 2,34 y 3,65 que marcan buen nivel de cumplimiento y son considerados importantes, pero no por ello debemos considerarlos como puntos fuertes o factores de calidad del centro educativo.

\section{ABOUT THE AUTHORS / SOBRE LOS AUTORES}

Jesús Miguel Muñoz Cantero (munoz@udc.es): . Es el autor de contacto para comunicaciones relacionadas con este artículo. Profesor Titular del área de MIDE, es director de la Unidad Técnica de Evaluación de la Universidad de A Coruña. Imparte docencia de Métodos y Técnicas de Evaluación de Centros en la titulación de Psicopedagogía y de Métodos de Investigación en Logopedia. Su trayectoria investigadora en los últimos años está centrada en la calidad y la evaluación de centros educativos. Buscar otros artículos de este autor en Scholar Google

Luz Sandra Casar Domínguez (sandracasar@edu.xunta.es): Doctora en Psicopedagogía, desarrolla su actividad investigadora en calidad y atención a la diversidad en centros no universitarios. Actualmente trabaja en la Xunta de Galicia. Buscar otros artículos de esta autora en Scholar Google Google

Eduardo Abalde Paz (abalde@udc.es): Catedrático del área de MIDE de la Universidad de A Coruña. Imparte su docencia en la titulación de Psicopedagogía en la materia de Métodos de Investigación en Educación. Desarrolla su investigación en evaluación de centros. Buscar otros artícu- 
Muñoz Cantero, J.M., Casar Domínguez, L. S. y Abalde Paz, E. (2007). El “contexto" y las "metas y objetivos" como elementos clave en la calidad de la atención a la diversidad en centros no universitarios.

RELIEVE, v. 13, n. 2, p. 235-261. http://www.uv.es/RELIEVE/v13n2/RELIEVEv13n2 6.htm

\section{ARTICLE RECORD / FICHA DEL ARTÍCULO}

\begin{tabular}{|c|c|}
\hline $\begin{array}{l}\text { Reference / } \\
\text { Referencia }\end{array}$ & $\begin{array}{l}\text { Muñoz Cantero, Jesús Miguel, Casar Domínguez, Luz Sandra, Abalde Paz, Eduardo (2007). El "contex- } \\
\text { to y las "metas y objetivos" como elementos clave en la calidad de la atención a la diversidad en centros } \\
\text { no universitarios. RELIEVE, v. 13, n. 2. http://www.uv.es/RELIEVE/v13n2/RELIEVEv13n2_6.htm. } \\
\text { Consultado en (poner fecha). }\end{array}$ \\
\hline Title / Título & $\begin{array}{l}\text { El "contexto y las "metas y objetivos" como elementos clave en la calidad de la atención a la diversidad } \\
\text { en centros no universitarios [The "context" and " goals and objectives" as key elements in quality in } \\
\text { attention to diversity in non-university education] }\end{array}$ \\
\hline $\begin{array}{l}\text { Authors / } \\
\text { Autores }\end{array}$ & Muñoz Cantero, Jesús Miguel, Casar Domínguez, Luz Sandra, Abalde Paz, Eduardo \\
\hline $\begin{array}{l}\text { Review / } \\
\text { Revista }\end{array}$ & Revista ELectrónica de Investigación y EValuación Educativa (RELIEVE), v. 13, n. 2 \\
\hline ISSN & $1134-4032$ \\
\hline $\begin{array}{l}\text { Publication } \\
\text { date / } \\
\text { Fecha de } \\
\text { publicación }\end{array}$ & $\begin{array}{l}2007 \text { (Reception Date: } 2007 \text { October 31; Approval Date: } 2007 \text { December 24; Publication Date: } \\
2007 \text { December 26) }\end{array}$ \\
\hline $\begin{array}{l}\text { Abstract / } \\
\text { Resumen }\end{array}$ & $\begin{array}{l}\text { Currently there is a growing interest in educational quality and attention to diversity. This paper pre- } \\
\text { sents an analysis of two important aspects: the "context" in which the school must develop its activity } \\
\text { and the "goals and objectives" that a school should respond to define quality for diversity. Analyze the } \\
\text { degree of importance for teachers and the level of compliance in the centers, from data obtained in two } \\
\text { of the questionnaires. The study was conducted with teachers in the province of A Coruna (Spain). } \\
\text { Overall, we can say that the issues explored are considered important by teachers as indicators of } \\
\text { quality. However, on the contrary, its level of compliance in the centers is medium or low, forcing es- } \\
\text { tablishing measures for improvement. } \\
\text { Actualmente existe un interés creciente por la calidad educativa y la atención a la diversidad. En este } \\
\text { trabajo se presenta un análisis de dos aspectos importantes: el "Contexto" en el que el centro debe de } \\
\text { desarrollar su actividad y las "metas y objetivos" que un centro educativo debe definir para dar una } \\
\text { respuesta de calidad a la diversidad. Analizaremos el grado de importancia para los profesores y el nivel } \\
\text { de cumplimiento en los centros, a partir de los datos obtenidos en dos cuestionarios. El estudio se reali- } \\
\text { zó con profesores de la provincia de A Coruña (España). En líneas generales podemos decir que los } \\
\text { aspectos analizados son considerados importantes por los profesores como indicadores de calidad. Sin } \\
\text { embargo, por el contrario, su nivel de cumplimiento en los centros es medio o bajo, lo que obliga a es- } \\
\text { tablecer medidas de mejora y correctores de estrategias. }\end{array}$ \\
\hline $\begin{array}{l}\text { Keywords / } \\
\text { Descriptores }\end{array}$ & $\begin{array}{l}\text { Quality, attention to diversity, inclusion, testing, non-university education. } \\
\text { Calidad, atención a la diversidad, inclusión, evaluación, educación no universitaria. }\end{array}$ \\
\hline $\begin{array}{l}\text { Institution / } \\
\text { Institución }\end{array}$ & Universidade da Coruña (España). \\
\hline $\begin{array}{l}\text { Publication } \\
\text { site / } \\
\text { Dirección }\end{array}$ & http://www.uv.es/RELIEVE \\
\hline $\begin{array}{l}\text { Language / } \\
\text { Idioma }\end{array}$ & Spanish (Title, abstract and keywords in English ) \\
\hline
\end{tabular}


Muñoz Cantero, J.M., Casar Domínguez, L. S. y Abalde Paz, E. (2007). El “contexto" y las "metas y objetivos" como elementos clave en la calidad de la atención a la diversidad en centros no universitarios.

RELIEVE, v. 13, n. 2, p. 235-261. http://www.uv.es/RELIEVE/v13n2/RELIEVEv13n2 6.htm

\section{Revista ELectrónica de Investigación y $\mathbf{E V}$ aluación Educativa}

\section{(RELIEVE)}

\section{[ ISSN: 1134-4032 ]}

(C) Copyright, RELIEVE. Reproduction and distribution of this articles it is authorized if the content is no modified and their origin is indicated (RELIEVE Journal, volume, number and electronic address of the document).

(C) Copyright, RELIEVE. Se autoriza la reproducción y distribución de este artículo siempre que no se modifique el contenido y se indique su origen (RELIEVE, volumen, número y dirección electrónica del documento). 